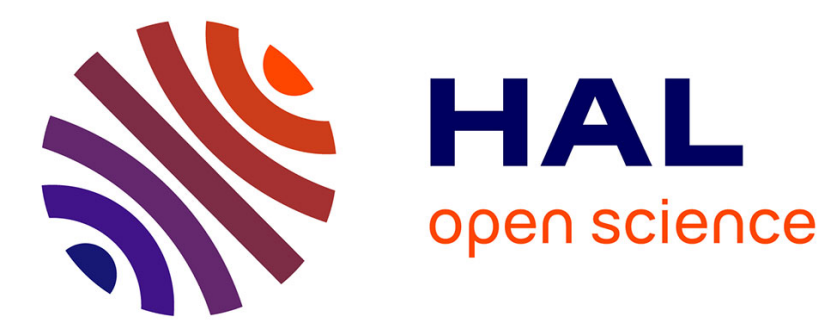

\title{
Natural Frequency Computation of Parallel Robots
}

\author{
Coralie Germain, Sébastien Briot, Stéphane Caro, Philippe Wenger
}

\section{To cite this version:}

Coralie Germain, Sébastien Briot, Stéphane Caro, Philippe Wenger. Natural Frequency Computation of Parallel Robots. Journal of Computational and Nonlinear Dynamics, 2015, 10 (2), 10.1115/1.4028573 . hal-01061512

\section{HAL Id: hal-01061512 https://hal.science/hal-01061512}

Submitted on 14 Aug 2018

HAL is a multi-disciplinary open access archive for the deposit and dissemination of scientific research documents, whether they are published or not. The documents may come from teaching and research institutions in France or abroad, or from public or private research centers.
L'archive ouverte pluridisciplinaire HAL, est destinée au dépôt et à la diffusion de documents scientifiques de niveau recherche, publiés ou non, émanant des établissements d'enseignement et de recherche français ou étrangers, des laboratoires publics ou privés. 


\title{
Natural Frequency Computation of Parallel Robots
}

\author{
Coralie Germain ${ }^{\dagger}$ Sébastien Briot ${ }^{\dagger *}$ \\ Stéphane Caro $^{\dagger} \quad$ Philippe Wenger ${ }^{\dagger}$ \\ †Institut de Recherche en Communications et Cybernétique de Nantes \\ IRCCyN, UMR CNRS 6597, Nantes, France \\ \{germain, briot, caro, wenger\}@irccyn.ec-nantes.fr \\ * Corresponding author \\ Tel.: +33 (0)2 40376958 \\ Fax: +33 (0)2 40376930
}

May 5, 2014

\begin{abstract}
The characterization of the elastodynamic behaviour and natural frequencies of parallel robots is a crucial point. Accurate elastodynamic models of parallel robots are useful at both their design and control stages in order to define their optimal dimensions and shapes while improving their vibratory behaviour.

Several methods exist to write the elastodynamic model of manipulators. However, those methods do not provide a straightforward way to write the Jacobian matrices related to the kinematic constraints of parallel manipulators. Therefore, the subject of this paper is about a systematic method for the determination of the mass and stiffness matrices of any parallel robot in any stationary configuration. The proposed method is used to express the mass and stiffness matrices of the NaVARo, a three-degree-of-freedom planar parallel robot with variable actuation schemes, developed at IRCCyN. Then, its natural frequencies are evaluated and compared with those obtained from both Cast3M software and experimentally.
\end{abstract}

\section{Introduction}

Parallel robots have been increasingly used in industry in the last few years for pick-and-place applications and high-speed machining $[1,2]$. This interest is mainly due to their high stiffness and good dynamic performance compared with their serial manipulator counterparts.

Having a good knowledge of the elastodynamic behaviour of a manipulator (especially its natural frequencies) is a crucial point. As a consequence, accurate elastodynamic models of 
parallel robots are useful at both their design $[3,4,5]$ and control $[6,7,8]$ stages in order to define their optimal dimensions and shapes while improving their vibratory behaviour.

Several models have been proposed and used in order to compute the natural frequencies of a mechanism. For instance, the following three methods are commonly used in the literature:

- Finite Element Analysis (FEA); the FEA method is proved to be the most accurate and reliable, since the links and joints are modeled with their true dimensions and shape $[9,10]$. However, its accuracy is limited by the mesh size and is usually used at the final design stage of the robot because the meshing is time-consuming.

- Matrix Structural Analysis $(M S A)$ method is a common technique in mechanical engineering $[4,11,12,13,14]$. It incorporates the main ideas of the $F E A$ method but operates with rather large flexible elements (beams, arcs, cables, and so on). As a result, the $M S A$ method is less time consuming than the FEA method, but requires good knowledge in FEA.

- Virtual joint method ( VJM ) [5, 15], which is also referred to as "lumped modeling", is based on the expansion of the traditional rigid model by adding virtual joints (localized springs), which describe the elastic deformations of the manipulator components (links, joints and actuators). Lumped modeling is simpler to use but is less accurate than $M S A$.

Some general methodologies dealing with $M S A$ or $V J M$ are presented in $[11,12,13,15]$. These methodologies can differ from the type of elements or flexible models used. Nevertheless, all of them require the determination of some Jacobian matrices in order to characterize kinematic dependencies in closed-loop mechanisms. The main drawback of such methodologies is that they are not specifically designed for parallel robots and they do not propose a systematic and straightforward way for computing the Jacobian matrices required for closing the loops.

An approach for the systematic computation of these matrices was proposed in [4]. However, this approach is complicated and the obtained results have not been verified either with a commercial software or experimentally. In [14], the authors proposed an interesting algorithm to study the elastodynamic behaviour of parallel robots that considers both the joint and link flexibilities. The way the authors close the robot loops and choose the independant coordinates is not straightforward, resulting in a potentially asymmetrical description of the leg variables.

This paper aims at developing a simple and straightforward procedure for the computation of mass and stiffness matrices of parallel robots in any stationary configuration. Indeed,

- a simple way to compute the Jacobian matrices required for closing the robot loops is presented, and

- the set of independent coordinates for parallel robots with a symmetrical arrangement are chosen cleverly.

Moreover, contrary to most of the existing methods, the proposed approach does not contain any numerical matrix inversion, which is better to avoid numerical issues that may lead to a loss in the result accuracy. The natural frequencies calculated using this procedure will be compared with simulation data obtained using a $F E A / M S A$ software and with experiments carried out on a parallel robot.

A methodology similar to the one presented in [16] to obtain the rigid dynamic model of a parallel robot is used. For a parallel robot composed of rigid or flexible links connected by passive 
or active joints, such as the one described in Fig. 1, the methodology is decomposed into two steps:

1. All closed-loops are virtually opened in such a way that the platform is virtually disassembled from the robot architecture (Fig. 1(b)). Each joint is virtually considered actuated so that the robot becomes a tree structure with a free body: the platform. The mass and stiffness matrices of the tree structure and the ones of the free platform are then computed;

2. The loops are closed using Jacobian matrices so that the mass and stiffness matrices of the actual parallel robot can be obtained.

The paper is organized as follows. In Section 2, a method used for the computation of the mass and stiffness matrices of one single flexible body using $M S A$ techniques is recalled. The mass and stiffness matrices of the virtual system composed of the tree structure and the free platform are introduced in Section 3. A straightforward way for the computation of the Jacobian matrices linking the Cartesian coordinates of each body to the generalized coordinates of the virtual system is proposed. Section 4 describes the proposed method to close the loops in order to obtain the mass and stiffness matrices of the actual parallel robot, that keeps a symmetrical description of the leg variables. The natural frequencies of the NaVARo $[17,18]$ are then evaluated in Section 5. NaVARo is a three-degree-of-freedom (dof) planar parallel manipulator with multiple actuation modes developed at IRCCyN. Moreover, the simulation results obtained with our approach are compared with both Cast3M software [19] and experimentations. Finally, some conclusions and future works are proposed in Section 6 .

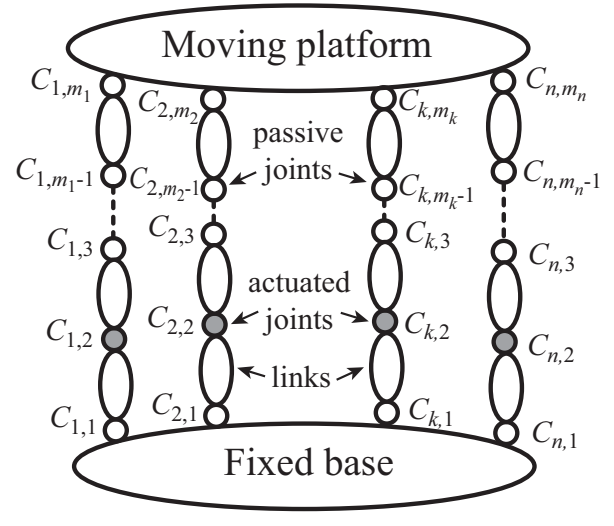

(a) Kinematic chain

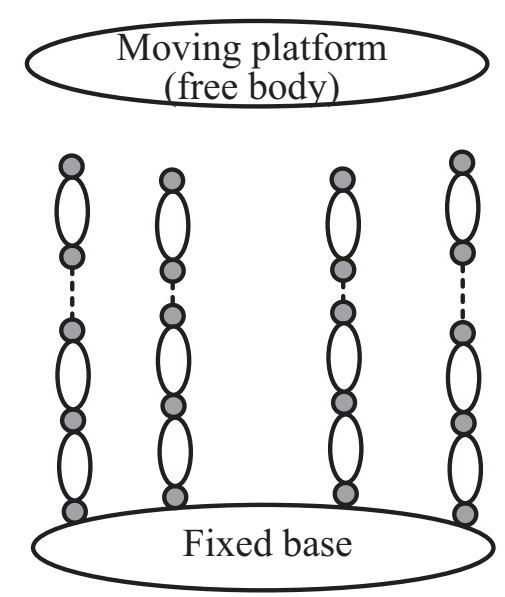

(b) Virtual tree structure

Figure 1: Schematic of a parallel robot for its dynamic modeling 


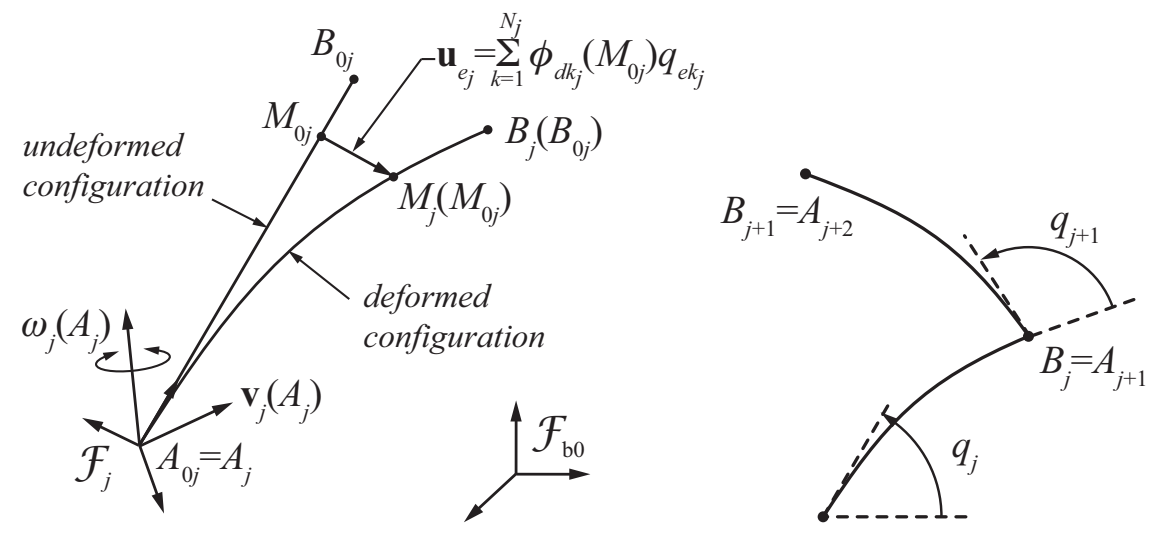

(a) Parameters of one flexible body $j$

(b) Assembly of two flexible bodies

Figure 2: Schematics of the flexible elements into consideration

\section{Computation of the Stiffness and Mass Matrices of a Flexible Body}

\subsection{Kinematics of a Flexible Free Body}

Let us consider body $j$ described in Fig. $2\left(\right.$ a) to which a local frame $\mathcal{F}_{j}$ is attached at point $A_{j}$. The flexible body is considered as the superposition of the undeformed configuration referred to as 0 , which corresponds to a rigid transformation of body $j$ described by variable $q_{j}$ and the deformed configuration related to the elastic deformation of the body. The translational velocity of any material point $M_{j}$ of this flexible body can be expressed as [20]:

$$
\mathbf{v}_{j}\left(M_{j}\right)=\mathbf{v}_{j}\left(A_{j}\right)+\boldsymbol{\omega}_{j}\left(A_{j}\right) \times \mathbf{r}_{j}\left(M_{j}\right)+\mathbf{v}_{e_{j}}\left(M_{j}\right)
$$

where $\mathbf{v}_{j}\left(A_{j}\right)$ and $\boldsymbol{\omega}_{j}\left(A_{j}\right)$ are the translational and rotational velocities of body $j$ expressed in frame $\mathcal{F}_{j}$, respectively, $\mathbf{r}_{j}\left(M_{j}\right)$ is the position vector of point $M_{j}$ (of the deformed body) expressed in frame $\mathcal{F}_{j}, \mathbf{v}_{e_{j}}\left(M_{j}\right)$ is the translational velocity due to the body elasticity that can be parameterized as truncated series of Rayleigh-Ritz shape functions:

$$
\mathbf{v}_{e_{j}}\left(M_{j}\right)=\boldsymbol{\Phi}_{d_{j}}\left(M_{0 j}\right) \dot{\mathbf{q}}_{e_{j}}
$$

with $\boldsymbol{\Phi}_{d_{j}}=\left[\phi_{d 1_{j}} \cdots \phi_{d N_{j_{j}}}\right]$ of size $\left(3 \times N_{j}\right), \phi_{d k_{j}}\left(M_{0 j}\right)$ being a three-dimensional vector containing the $k$-th shape functions for the displacement of the flexible body at point $M_{0 j}$, and $\dot{\mathbf{q}}_{e_{j}}=\left[\dot{q}_{e 1_{j}} \cdots \dot{q}_{e N_{j_{j}}}\right]^{T}, \dot{q}_{e k_{j}}$ being the $k$-th elastic generalized velocity of body $j$ and $N_{j}$ the number of considered shape functions. It should be noted that vector $\mathbf{r}_{j}\left(M_{j}\right)$ in (1) can be expressed as:

$$
\mathbf{r}_{j}\left(M_{j}\right)=\mathbf{r}_{j}\left(M_{0 j}\right)+\mathbf{u}_{e_{j}}\left(M_{0 j}\right)
$$

where $\mathbf{r}_{j}\left(M_{0_{j}}\right)$ is the position vector of point $M_{0 j}$ expressed in frame $\mathcal{F}_{j}$,

$$
\mathbf{u}_{e_{j}}\left(M_{0 j}\right)=\boldsymbol{\Phi}_{d_{j}}\left(M_{0 j}\right) \mathbf{q}_{e_{j}}
$$


is the elastic displacement that transforms $M_{0 j}$ to $M_{j}$ and $\mathbf{q}_{e_{j}}=\left[q_{e 1_{j}} \cdots q_{e N_{j_{j}}}\right]^{T}$ is the vector of elastic generalized coordinates of body $j$.

Equation (1) can thus be written the following matrix form:

$$
\mathbf{v}_{j}\left(M_{j}\right)=\left[\begin{array}{lll}
\mathbf{I}_{3} & {\left[\mathbf{r}_{j}\left(M_{j}\right)\right]_{\times}^{T}} & \boldsymbol{\Phi}_{d_{j}}\left(M_{0 j}\right)
\end{array}\right]\left[\begin{array}{c}
\mathbf{v}_{j}\left(A_{j}\right) \\
\boldsymbol{\omega}_{j}\left(A_{j}\right) \\
\dot{\mathbf{q}}_{e_{j}}
\end{array}\right]
$$

where $\mathbf{I}_{3}$ is the $(3 \times 3)$ identity matrix and $\left[\mathbf{r}_{j}\left(M_{j}\right)\right]_{\times}$is the cross-product matrix of vector $\mathbf{r}_{j}\left(M_{j}\right)$.

Equations (1) to (5) define the kinematic model of flexible free body $j$. This model is thus parameterized by the following set of variables:

- $\mathbf{v}_{j}\left(A_{j}\right)$ and $\boldsymbol{\omega}_{j}\left(A_{j}\right)$ : Cartesian velocities (Euler variables) characterizing the rigid displacement of body $j$ expressed in frame $\mathcal{F}_{j}$;

- $\mathbf{q}_{e_{j}}$ : generalized coordinates (Lagrange variables) characterizing the elastic displacements of body $j$.

It should be mentioned that this description can be applied to both robot links and joints, as long as all the shape functions can be defined.

\subsection{Kinetic Energy of a Flexible Free Body}

The kinetic energy of body $j$ (denoted as $\Sigma_{j}$ in the following integral) is given by:

$$
T_{j}=\frac{1}{2} \int_{\Sigma_{j}} \mathbf{v}_{j}\left(M_{j}\right)^{T} \mathbf{v}_{j}\left(M_{j}\right) d m=\frac{\rho_{j}}{2} \int_{V_{j}} \mathbf{v}_{j}\left(M_{j}\right)^{T} \mathbf{v}_{j}\left(M_{j}\right) d V
$$

where $\rho_{j}$ is the material density and $V_{j}$ is the body volume.

Introducing (5) into (6) leads to:

$$
\begin{aligned}
T_{j} & =\frac{\rho_{j}}{2} \int_{V_{j}}\left[\begin{array}{c}
\mathbf{v}_{j}\left(A_{j}\right) \\
\boldsymbol{\omega}_{j}\left(A_{j}\right) \\
\dot{\mathbf{q}}_{e_{j}}
\end{array}\right]^{T}\left[\begin{array}{c}
\mathbf{I}_{3} \\
{\left[\mathbf{r}_{j}\left(M_{j}\right)\right]_{\times}} \\
\mathbf{\Phi}_{d_{j}}\left(M_{0 j}\right)^{T}
\end{array}\right]\left[\begin{array}{lll}
\mathbf{I}_{3} & {\left[\mathbf{r}_{j}\left(M_{j}\right)\right]_{\times}^{T}} & \boldsymbol{\Phi}_{d_{j}}\left(M_{0 j}\right)
\end{array}\right]\left[\begin{array}{c}
\mathbf{v}_{j}\left(A_{j}\right) \\
\boldsymbol{\omega}_{j}\left(A_{j}\right) \\
\dot{\mathbf{q}}_{e_{j}}
\end{array}\right] d V \\
& =\frac{1}{2}\left[\begin{array}{c}
\mathbf{v}_{j}\left(A_{j}\right) \\
\boldsymbol{\omega}_{j}\left(A_{j}\right) \\
\dot{\mathbf{q}}_{e_{j}}
\end{array}\right]^{T} \mathbf{M}_{j}\left[\begin{array}{c}
\mathbf{v}_{j}\left(A_{j}\right) \\
\boldsymbol{\omega}_{j}\left(A_{j}\right) \\
\dot{\mathbf{q}}_{e_{j}}
\end{array}\right]
\end{aligned}
$$

where

$$
\mathbf{M}_{j}=\rho_{j} \int_{V_{j}}\left[\begin{array}{ccc}
\mathbf{I}_{3} & {\left[\mathbf{r}_{j}\left(M_{j}\right)\right]_{\times}^{T}} & \boldsymbol{\Phi}_{d_{j}}\left(M_{0 j}\right) \\
{\left[\mathbf{r}_{j}\left(M_{j}\right)\right]_{\times}} & {\left[\mathbf{r}_{j}\left(M_{j}\right)\right]_{\times}\left[\mathbf{r}_{j}\left(M_{j}\right)\right]_{\times}^{T}} & {\left[\mathbf{r}_{j}\left(M_{j}\right)\right]_{\times} \boldsymbol{\Phi}_{d_{j}}\left(M_{0 j}\right)} \\
\boldsymbol{\Phi}_{d_{j}}\left(M_{0 j}\right)^{T} & \boldsymbol{\Phi}_{d_{j}}\left(M_{0 j}\right)^{T}\left[\mathbf{r}_{j}\left(M_{j}\right)\right]_{\times}^{T} & \boldsymbol{\Phi}_{d_{j}}\left(M_{0 j}\right)^{T} \boldsymbol{\Phi}_{d_{j}}\left(M_{0 j}\right)
\end{array}\right] d V
$$

$\mathbf{M}_{j}$ is the mass matrix of body $j$ expressed in its frame $\mathcal{F}_{j}$. 


\subsection{Elastic Potential Energy and Stiffness Matrix of a Flexible Free Body}

The elastic potential energy of any body is given by [11]:

$$
V_{e_{j}}=\frac{1}{2} \int_{V_{j}} \boldsymbol{\sigma}_{j}^{T} \mathbf{I}_{t} \boldsymbol{\epsilon}_{j} d V
$$

where $\boldsymbol{\sigma}_{j}$ and $\boldsymbol{\epsilon}_{j}$ are the six-dimensional stress and strain vectors due to the small elastic displacement $\mathbf{u}_{e_{j}}\left(M_{0 j}\right)$ in body $j . \quad \mathbf{I}_{t}$ is a $(6 \times 6)$ diagonal matrix. The first three diagonal terms are equal to one, whereas the last three diagonal terms are equal to two, because of the two multipliers associated with the shear strains [11]. The strain vector is defined as $\boldsymbol{\epsilon}_{j}=\left[\begin{array}{llllll}\epsilon_{j_{11}} & \epsilon_{j_{22}} & \epsilon_{j_{33}} & \epsilon_{j_{12}} & \epsilon_{j_{13}} & \epsilon_{j_{23}}\end{array}\right]^{T}$, where:

$$
\left[\begin{array}{ccc}
\epsilon_{j_{11}} & \epsilon_{j_{12}} & \epsilon_{j_{13}} \\
\epsilon_{j_{12}} & \epsilon_{j_{22}} & \epsilon_{j_{23}} \\
\epsilon_{j_{13}} & \epsilon_{j_{23}} & \epsilon_{j_{33}}
\end{array}\right]=\frac{1}{2}\left(\nabla \mathbf{u}_{e_{j}}\left(M_{0 j}\right)+\left(\nabla \mathbf{u}_{e_{j}}\left(M_{0 j}\right)\right)^{T}\right)
$$

with

$$
\begin{aligned}
\nabla \mathbf{u}_{e_{j}}\left(M_{0 j}\right)= & {\left[\begin{array}{lll}
\frac{\partial \mathbf{u}_{e_{j}}}{\partial x}\left(M_{0 j}\right) & \frac{\partial \mathbf{u}_{e_{j}}}{\partial y}\left(M_{0 j}\right) & \frac{\partial \mathbf{u}_{e_{j}}}{\partial z}\left(M_{0 j}\right)
\end{array}\right] } \\
= & {\left[\begin{array}{lll}
\frac{\partial \boldsymbol{\Phi}_{d_{j}}^{1}}{\partial x}\left(M_{0 j}\right) \mathbf{q}_{e_{j}} & \frac{\partial \boldsymbol{\Phi}_{d_{j}}^{1}}{\partial y}\left(M_{0 j}\right) \mathbf{q}_{e_{j}} & \frac{\partial \boldsymbol{\Phi}_{d_{j}}^{1}}{\partial z}\left(M_{0 j}\right) \mathbf{q}_{e_{j}} \\
\frac{\partial \boldsymbol{\Phi}_{d_{j}}^{2}}{\partial x}\left(M_{0 j}\right) \mathbf{q}_{e_{j}} & \frac{\partial \boldsymbol{\Phi}_{d_{j}}^{2}}{\partial y}\left(M_{0 j}\right) \mathbf{q}_{e_{j}} & \frac{\partial \boldsymbol{\Phi}_{d_{j}}^{2}}{\partial z}\left(M_{0 j}\right) \mathbf{q}_{e_{j}} \\
\frac{\partial \boldsymbol{\Phi}_{d_{j}}^{3}}{\partial x}\left(M_{0 j}\right) \mathbf{q}_{e_{j}} & \frac{\partial \boldsymbol{\Phi}_{d_{j}}^{3}}{\partial y}\left(M_{0 j}\right) \mathbf{q}_{e_{j}} & \frac{\partial \boldsymbol{\Phi}_{d_{j}}^{3}}{\partial z}\left(M_{0 j}\right) \mathbf{q}_{e_{j}}
\end{array}\right] }
\end{aligned}
$$

where $\boldsymbol{\Phi}_{d_{j}}^{k}$ corresponds to the $k$-th line of matrix $\boldsymbol{\Phi}_{d_{j}}, k=1,2,3$. As a result,

$$
\boldsymbol{\epsilon}_{j}=\left[\begin{array}{c}
\frac{\partial \boldsymbol{\Phi}_{d_{j}}^{1}}{\partial x}\left(M_{0 j}\right) \\
\frac{\partial \boldsymbol{\Phi}_{d_{j}}^{2}}{\partial y}\left(M_{0 j}\right) \\
\frac{\partial \boldsymbol{\Phi}_{d_{j}}^{3}}{\partial z}\left(M_{0 j}\right) \\
\frac{1}{2}\left(\frac{\partial \boldsymbol{\Phi}_{d_{j}}^{1}}{\partial y}\left(M_{0 j}\right)+\frac{\partial \boldsymbol{\Phi}_{d_{j}}^{2}}{\partial x}\left(M_{0 j}\right)\right) \\
\frac{1}{2}\left(\frac{\partial \boldsymbol{\Phi}_{d_{j}}^{1}}{\partial z}\left(M_{0 j}\right)+\frac{\partial \boldsymbol{\Phi}_{d_{j}}^{3}}{\partial x}\left(M_{0 j}\right)\right) \\
\frac{1}{2}\left(\frac{\partial \boldsymbol{\Phi}_{d_{j}}^{2}}{\partial z}\left(M_{0 j}\right)+\frac{\partial \boldsymbol{\Phi}_{d_{j}}^{3}}{\partial y}\left(M_{0 j}\right)\right)
\end{array}\right] \mathbf{q}_{e_{j}}=\boldsymbol{\Phi}_{\epsilon_{j}} \mathbf{q}_{e_{j}}
$$

The stress vector is expressed as:

$$
\boldsymbol{\sigma}_{j}=\left[\begin{array}{llllll}
\sigma_{j_{11}} & \sigma_{j_{22}} & \sigma_{j_{33}} & \sigma_{j_{12}} & \sigma_{j_{13}} & \sigma_{j_{23}}
\end{array}\right]^{T}=\mathbf{E}_{j} \boldsymbol{\epsilon}_{j}
$$


where matrix $\mathbf{E}_{j}$ is given by the Hooke's law [11].

Thus, introducing (12) and (13) into (9) leads to:

$$
V_{e_{j}}=\frac{1}{2} \mathbf{q}_{e_{j}}^{T} \mathbf{K}_{j} \mathbf{q}_{e_{j}}
$$

where $\mathbf{K}_{j}$ is the stiffness matrix of body $j$ and takes the form:

$$
\mathbf{K}_{j}=\int_{V_{j}} \boldsymbol{\Phi}_{\epsilon_{j}}^{T} \mathbf{E}_{j}^{T} \mathbf{I}_{t} \boldsymbol{\Phi}_{\epsilon_{j}} d V
$$

\subsection{Case of the Elastic Beam: the Bernoulli Model}

The computation of the mass and stiffness matrices of 3D beams is useful for the elastodynamic modeling of parallel manipulators.

The Bernoulli model describes the beam deformation under the assumption that the shear effect is negligible, that the cross-sections remain perpendicular to the neutral axis and that the rotational inertia of sections is assumed to be zero [21]. With such a model, the 3D beam deformation $\mathbf{u}_{e_{j}}\left(M_{0 j}\right)$ (see Section 2.1) can be characterized with the six shape functions $\phi_{d x_{j}}$, $\phi_{d y_{j}}, \phi_{d z_{j}}, \phi_{r x_{j}}, \phi_{r y_{j}}$ and $\phi_{r z_{j}}$, i.e. $N_{j}=6$, defined as:

$$
\begin{aligned}
\phi_{d x_{j}} & =\left[\begin{array}{llllll}
\xi & 0 & 0 & 0 & 0 & 0
\end{array}\right] \\
\phi_{d y_{j}} & =\left[\begin{array}{llllll}
0 & 3 \xi^{2}-2 \xi^{3} & 0 & 0 & 0 & l_{j}\left(\xi^{3}-\xi^{2}\right)
\end{array}\right] \\
\phi_{d z_{j}} & =\left[\begin{array}{llllll}
0 & 0 & 3 \xi^{2}-2 \xi^{3} & 0 & -l_{j}\left(\xi^{3}-\xi^{2}\right) & 0
\end{array}\right] \\
\boldsymbol{\phi}_{r x_{j}} & =\left[\begin{array}{llllll}
0 & 0 & 0 & \xi & 0 & 0
\end{array}\right] \\
\boldsymbol{\phi}_{r y_{j}} & =\left[\begin{array}{llllll}
0 & 0 & -6\left(\xi-\xi^{2}\right) / l_{j} & 0 & 3 \xi^{2}-2 \xi & 0
\end{array}\right] \\
\phi_{r z_{j}} & =\left[\begin{array}{lllllll}
0 & 6\left(\xi-\xi^{2}\right) / l_{j} & 0 & 0 & 0 & 3 \xi^{2}-2 \xi
\end{array}\right]
\end{aligned}
$$

where $\xi=x / l_{j}$ and $l_{j}$ is the beam length.

$x, y$ and $z$ denote the Cartesian coordinates of point $M_{0 j}$ expressed in the local frame $\mathcal{F}_{j}$ and $\boldsymbol{\Phi}_{d_{j}}\left(M_{0 j}\right)$ is a $(3 \times 6)$ matrix that takes the form:

$$
\boldsymbol{\Phi}_{d_{j}}\left(M_{0 j}\right)=\left[\begin{array}{c}
\boldsymbol{\phi}_{d x_{j}}-y \boldsymbol{\phi}_{r z_{j}}+z \boldsymbol{\phi}_{r y_{j}} \\
\boldsymbol{\phi}_{d y_{j}}-z \boldsymbol{\phi}_{r x_{j}} \\
\boldsymbol{\phi}_{d z_{j}}+y \boldsymbol{\phi}_{r x_{j}}
\end{array}\right]
$$

Moreover, in the beam model, it is assumed that

$$
\begin{aligned}
\sigma_{j_{22}} & =\sigma_{j_{33}}=\sigma_{j_{23}}=0 \\
\epsilon_{j_{22}} & =\epsilon_{j_{33}}=\epsilon_{j_{23}}=0 \\
\sigma_{j_{11}} & =E_{j} \epsilon_{j_{11}} \\
\sigma_{j_{12}} & =G_{j} \epsilon_{j_{12}} \\
\sigma_{j_{13}} & =G_{j} \epsilon_{j_{13}}
\end{aligned}
$$

where $E_{j}$ is the Young modulus of body $j$ and $G_{j}$ is its shear modulus. 
Introducing (16a) to (22) into (8) and (15) for $\mathbf{q}_{e_{j}}=\mathbf{0}$, the stiffness matrix of body $j$ takes the form:

$$
\mathbf{K}_{j}=\frac{1}{l_{j}^{3}}\left[\begin{array}{cccccc}
E_{j} A_{j} l_{j}^{2} & 0 & 0 & 0 & 0 & 0 \\
0 & 12 E_{j} I_{z_{j}} & 0 & 0 & 0 & -6 E_{j} I_{z_{j}} l_{j} \\
0 & 0 & 12 E_{j} I_{y_{j}} & 0 & 6 E_{j} I_{y_{j}} l_{j} & 0 \\
0 & 0 & 0 & I_{0_{j}} G_{j} l_{j}^{2} & 0 & 0 \\
0 & 0 & 6 E_{j} I_{y_{j}} l_{j} & 0 & 4 E_{j} I_{y_{j}} l_{j}^{2} & 0 \\
0 & -6 E_{j} I_{z_{j}} l_{j} & 0 & 0 & 0 & 4 E_{j} I_{z_{j}} l_{j}^{2}
\end{array}\right]
$$

where $A_{j}$ is the beam cross-section area, $I_{y_{j}}$ and $I_{z_{j}}$ are the second moments of area around axes $y$ and $z$ of the local frame, $I_{0_{j}}$ is the torsion constant.

Similarly, the mass matrix of body $j$ is expressed as:

$$
\mathbf{M}_{j}=\left[\begin{array}{ll}
\mathbf{M}_{11_{j}} & \mathbf{M}_{12_{j}} \\
\mathbf{M}_{12_{j}}^{T} & \mathbf{M}_{22_{j}}
\end{array}\right]
$$

where

$$
\begin{aligned}
& \mathbf{M}_{11_{j}}=\left[\begin{array}{cccccc}
m_{j} & 0 & 0 & 0 & m_{j} z_{G_{j}} & -m_{j} y_{G_{j}} \\
0 & m_{j} & 0 & -m_{j} z_{G_{j}} & 0 & m_{j} x_{G_{j}} \\
0 & 0 & m_{j} & m_{j} y_{G_{j}} & -m_{j} x_{G_{j}} & 0 \\
0 & -m_{j} z_{G_{j}} & m_{j} y_{G_{j}} & J_{x x_{j}} & J_{x y_{j}} & J_{x z_{j}} \\
m_{j} z_{G_{j}} & 0 & -m_{j} x_{G_{j}} & J_{x y_{j}} & J_{y y_{j}} & J_{y z_{j}} \\
-m_{j} y_{G_{j}} & m_{j} x_{G_{j}} & 0 & J_{x z_{j}} & J_{y z_{j}} & J_{z z_{j}}
\end{array}\right] \\
& \mathbf{M}_{12_{j}}=\left[\begin{array}{cccccc}
\frac{m_{j}}{2} & 0 & 0 & 0 & 0 & 0 \\
0 & \frac{m_{j}}{2} & 0 & 0 & 0 & \frac{m_{j} l_{j}}{12} \\
0 & 0 & \frac{m_{j}}{2} & 0 & \frac{m_{j} l_{j}}{12} & 0 \\
0 & 0 & 0 & \frac{\rho_{j} l_{j} I_{p_{j}}}{2} & 0 & 0 \\
0 & 0 & -\rho_{j} I_{y_{j}}-\frac{7 m_{j} l_{j}}{20} & 0 & -\frac{m_{j} l_{j}^{2}}{20} & 0 \\
0 & \rho_{j} I_{z_{j}}+\frac{7 m_{j} l_{j}}{20} & 0 & 0 & 0 & -\frac{m_{j} l_{j}^{2}}{20}
\end{array}\right] \\
& \mathbf{M}_{22_{j}}=\left[\begin{array}{cccccc}
\frac{m_{j}}{3} & 0 & 0 & 0 & 0 & 0 \\
0 & \frac{13 m_{j}}{35}+\frac{6 \rho_{j} I_{z_{j}}}{5 l_{j}} & 0 & 0 & 0 & -\frac{11 m_{j} l_{j}+21 \rho_{j} I_{z_{j}}}{210} \\
0 & 0 & \frac{13 m_{j}}{35}+\frac{6 \rho_{j} I_{y_{j}}}{5 l_{j}} & 0 & \frac{11 m_{j} l_{j}+21 \rho_{j} I_{y_{j}}}{210} & 0 \\
0 & 0 & 0 & \frac{\rho_{j} l_{j} I_{p_{j}}}{3} & 0 & 0 \\
0 & 0 & \frac{11 m_{j} l_{j}+21 \rho_{j} I_{y_{j}}}{210} & 0 & \frac{m_{j} l_{j}^{2}+14 \rho_{j} I_{y_{j}} l_{j}}{105} & 0 \\
0 & -\frac{11 m_{j} l_{j}+21 \rho_{j} I_{z_{j}}}{210} & 0 & 0 & 0 & \frac{m_{j} l_{j}^{2}+14 \rho_{j} I_{z_{j}} l_{j}}{105}
\end{array}\right]
\end{aligned}
$$

and

- $x_{G_{j}}, y_{G_{j}}$ and $z_{G_{j}}$ are the Cartesian coordinates of the center of mass of body $j$ expressed in frame $\mathcal{F}_{j}$; 
- $J_{x x_{j}}, J_{y y_{j}}, J_{z z_{j}}, J_{x y_{j}}, J_{x z_{j}}$ and $J_{y z_{j}}$ are the terms of the rigid inertia matrix expressed at the origin of frame $\mathcal{F}_{j}$;

- $I_{p_{j}}=I_{y_{j}}+I_{z_{j}}$ is the polar moment of inertia.

\section{Computation of the Stiffness and Mass Matrices of a Tree Structure Robot}

Let us consider a parallel robot composed of one rigid fixed base (denoted as element 0), one rigid moving platform and $n$ legs, each leg being a serial kinematic chain composed of $m_{i}-1$ elements $^{1}$ connected by $m_{i}$ joints (revolute, prismatic or fixed joints - $i=1, \ldots, n$ ) located at points $C_{i k}\left(k=1, \ldots, m_{i}\right.$ - Fig. 1(a)). The $j$-th element of the $i$-th leg is denoted by $i j$. The nominal values of the actuated variables (of the passive variables, resp.) are denoted by $\mathbf{q}_{a}\left(\mathbf{q}_{p}\right.$, resp.). The nominal values of the Cartesian coordinates of the platform are denoted by $\mathbf{x}_{p}$. The dimension $n_{a}$ of vector $\mathbf{q}_{a}$ must be greater than or equal to the number of degrees of freedom of the parallel robot. Under the assumption of an elastic deformation, the variations in those variables are denoted by $\delta \mathbf{q}_{a}, \delta \mathbf{q}_{p}$ and $\delta \mathbf{x}_{p}$, respectively.

The number of shape functions for the element $i j$ is denoted by $N_{i j}\left(j=1, \ldots, m_{i}-1\right)$. As a result, the dimension $n_{e}$ of the vector of elastic variables $\mathbf{q}_{e}$ is equal to $\sum_{i=1}^{n} \sum_{j=1}^{m_{i}-1} N_{i j}$.

Thus, the vector of generalized coordinates of the tree-structure is given by $\mathbf{q}_{t}=\left[\mathbf{q}_{t_{1}}^{T} \cdots \mathbf{q}_{t_{n}}^{T}\right]^{T}$, where $\mathbf{q}_{t_{i}}=\left[\delta \mathbf{q}_{a_{i}}^{T} \delta \mathbf{q}_{p_{i}}^{T} \mathbf{q}_{e_{i}}^{T}\right]^{T} . \delta \mathbf{q}_{a_{i}}, \delta \mathbf{q}_{p_{i}}$ and $\mathbf{q}_{e_{i}}$ are the vectors of the actuated, passive and elastic generalized coordinates for the $i$-th leg.

\subsection{Relationships between the Generalized Coordinates of a Flexible Body and the Generalized Coordinates of the Tree Structure}

The generalized velocities $\mathbf{v}_{i j}\left(A_{i j}\right), \boldsymbol{\omega}_{i j}\left(A_{i j}\right)$ and $\dot{\mathbf{q}}_{e_{i j}}$ of body $i j$ are linked to the generalized velocities $^{2} \dot{\mathbf{q}}_{t}$ by the relations:

$$
\left[\begin{array}{c}
\mathbf{v}_{i j}\left(A_{i j}\right) \\
\boldsymbol{\omega}_{i j}\left(A_{i j}\right)
\end{array}\right]=\mathbf{J}_{v_{i j}}^{i} \dot{\mathbf{q}}_{t_{i}}=\mathbf{J}_{v_{i j}} \dot{\mathbf{q}}_{t}
$$

with

$$
\begin{aligned}
\mathbf{J}_{v_{i j}}= & {\left[\begin{array}{ccccc}
\mathbf{0} & \cdots & \mathbf{J}_{v_{i j}}^{i} & \cdots & \mathbf{0}
\end{array}\right] } \\
\dot{\mathbf{q}}_{t}= & {\left[\begin{array}{c}
\dot{\mathbf{q}}_{t_{1}} \\
\vdots \\
\dot{\mathbf{q}}_{t_{i}} \\
\vdots \\
\dot{\mathbf{q}}_{t_{n}}
\end{array}\right] }
\end{aligned}
$$

\footnotetext{
${ }^{1}$ Note that each robot link can be composed of one element or several elements.

${ }^{2}$ It is assumed that the generalized velocities are equal to $\frac{d}{d t}\left(\delta \mathbf{q}_{t}\right)=\dot{\mathbf{q}}_{t}$
} 
and

$$
\dot{\mathbf{q}}_{e_{i j}}=\mathbf{J}_{e_{i j}} \dot{\mathbf{q}}_{t}
$$

The foregoing equations can be rewritten in the following compact form:

$$
\left[\begin{array}{c}
\mathbf{v}_{i j}\left(A_{i j}\right) \\
\boldsymbol{\omega}_{i j}\left(A_{i j}\right) \\
\dot{\mathbf{q}}_{e_{i j}}
\end{array}\right]=\mathbf{J}_{i j} \dot{\mathbf{q}}_{t}
$$

with

$$
\mathbf{J}_{i j}=\left[\begin{array}{l}
\mathbf{J}_{v_{i j}} \\
\mathbf{J}_{e_{i j}}
\end{array}\right]
$$

$\mathbf{J}_{i j}$ is the Jacobian matrix mapping the generalized velocities $\dot{\mathbf{q}}_{t}$ of the tree structure into the generalized velocities of the element $i j . \mathbf{J}_{v_{i j}}$ is the Jacobian matrix mapping $\dot{\mathbf{q}}_{t}$ into the twist of the local frame attached to element $i j . \mathbf{J}_{e_{i j}}$ is a matrix composed of 0 and 1 terms that is used to extract vector $\dot{\mathbf{q}}_{e_{i j}}$ from vector $\dot{\mathbf{q}}_{t}$.

Matrix $\mathbf{J}_{v_{i j}}$ is expressed thanks to a generic and systematic approach described thereafter and derived from [22].

Let us first compute the homogeneous transformation matrix $\mathbf{T}_{i j}$ that defines the location and orientation of the local frame attached to the element $i j$. By definition, this element is located in the leg $i$ and is preceded by $j-1$ elements, each element $i k(k=1, \ldots, j)$ being linked to another by a joint (revolute, prismatic or fixed) described by its nominal coordinate $q_{i k}$ (Fig. 1(b)). Thus, matrix $\mathbf{T}_{i j}$ is defined by:

$$
\mathbf{T}_{i j}=\mathbf{T}_{b a s e}^{i}\left(\prod_{k=1}^{j-1}\left(\mathbf{V}_{a}\left(q_{i k}\right) \mathbf{T}_{e l t}^{i k} \mathbf{V}_{e}\left(\mathbf{q}_{e_{i k}}\right)\right)\right) \mathbf{V}_{a}\left(q_{i j}\right) \mathbf{T}_{e n d}^{i j}
$$

where

- $\mathbf{T}_{\text {base }}^{i}$ denotes the rigid transformation between the global base frame and the frame attached to $i$-th leg;

- The matrix function $\mathbf{V}_{a}($.$) is a transformation matrix corresponding to an elementary ro-$ tation or an elementary translation;

- $\mathbf{T}_{\text {elt }}^{i k}$ denotes the rigid transformation matrix between the frame attached to element $i k$ and the frame attached to element $i, k+1$ in the undeformed case;

- The matrix function $\mathbf{V}_{e}($.$) represents the translations and rotations due to the deformations$ of the flexible link (if the element is rigid, this matrix will be the identity matrix $\mathbf{q}_{e_{i k}}=\mathbf{0}$ in this case);

- $\mathbf{T}_{\text {end }}^{i j}$ is a matrix that allows the rotational part of $\mathbf{T}_{i j}$ to be equal to the identity matrix.

Then, let us gather all variables introduced in (33) into vector

$$
\mathbf{q}_{t_{i j}}=\left[q_{i 1} \mathbf{q}_{e_{i 1}}^{T} \cdots \mathbf{q}_{e_{i, j-1}}^{T} q_{i j}\right]^{T}
$$


and assume that for the $l$-th variable $q_{t_{i j l}}$ of vector $\mathbf{q}_{t_{i j}}$, (33) is rewritten as:

$$
\mathbf{T}_{i j}=\mathbf{H}_{i j l}^{R} \mathbf{V}_{i j l}\left(q_{t_{i j l}}\right) \mathbf{H}_{i j l}^{L}
$$

where the first and the third multipliers are constant homogeneous matrices while the second multiplier is either an elementary translation matrix or an elementary rotation matrix. Then the partial derivative of the homogeneous matrix $\mathbf{T}_{i j}$ with respect to $q_{t_{i j l}}$ at the configuration $q_{t_{i j l}}^{\text {nom }}$ $\left(q_{t_{i j l}}^{\text {nom }}=0\right.$ for an elastic variable and may not vanish for a joint variable $)$ can be computed from a similar product where the internal term is replaced by the matrix $\mathbf{V}_{i j l}^{d}()=.\partial \mathbf{V}_{i j l}\left(q_{t_{i j l}}\right) / \partial q_{t_{i j l}}$ that takes a simple analytical form. For instance, for elementary translations and rotations about the $x$ axis, these derivatives take the form:

$$
\begin{aligned}
\mathbf{V}_{i j l}^{d}\left(q_{t_{i j l}}\right) & =\left[\begin{array}{llll}
0 & 0 & 0 & 1 \\
0 & 0 & 0 & 0 \\
0 & 0 & 0 & 0 \\
0 & 0 & 0 & 0
\end{array}\right] \text { for a translation along } x \text { axis } \\
\mathbf{V}_{i j l}^{d}\left(q_{t_{i j l}}\right) & =\left[\begin{array}{cccc}
0 & 0 & 0 & 0 \\
0 & -\sin \left(q_{t_{i j l}}\right) & -\cos \left(q_{t_{i j l}}\right) & 0 \\
0 & \cos \left(q_{t_{i j l}}\right) & -\sin \left(q_{t_{i j l}}\right) & 0 \\
0 & 0 & 0 & 0
\end{array}\right] \text { for a rotation about } x \text { axis }
\end{aligned}
$$

For the elementary translations and rotations about the other axes, the matrices $\mathbf{V}_{i j l}^{d}($.$) can be$ obtained similarly.

Only small displacements occur in the determination of the natural frequencies. Therefore, the derivative of the homogeneous matrix $\mathbf{T}_{i j}^{d}=\partial \mathbf{T}_{i j} / \partial q_{t_{i j l}}=\mathbf{H}_{i j l}^{R} \mathbf{V}_{i j l}^{d}\left(q_{t_{i j l}}\right) \mathbf{H}_{i j l}^{L}$ can be expressed as:

$$
\mathbf{T}_{i j}^{d}=\left[\begin{array}{cccc}
0 & -\gamma_{z_{i j l}}^{d} & \gamma_{y_{i j l}}^{d} & \lambda_{x_{i j l}}^{d} \\
\gamma_{z_{i j l}}^{d} & 0 & -\gamma_{x_{i j l}}^{d} & \lambda_{y_{i j l}}^{d} \\
-\gamma_{y_{i j l}}^{d} & \gamma_{x_{i j l}}^{d} & 0 & \lambda_{z_{i j l}}^{d} \\
0 & 0 & 0 & 0
\end{array}\right]
$$

It is noteworthy that vector ${ }^{0} \mathbf{j}_{v_{i j}}^{q_{t_{i j l}}}=\left[\lambda_{x_{i j l}}^{d} \lambda_{y_{i j l}}^{d} \lambda_{z_{i j l}}^{d} \gamma_{x_{i j l}}^{d} \gamma_{y_{i j l}}^{d} \gamma_{z_{i j l}}^{d}\right]^{T}$, which can be obtained by extracting the terms of matrix $\mathbf{T}_{i j}^{d}$ defined in (37) is the column of the Jacobian matrix, corresponding to variable $\dot{q}_{i j l}$. The latter maps vector $\mathbf{q}_{t}$ into the velocity of the local frame attached to body $i j$ expressed in the global frame [22]. Let ${ }^{i j} \overline{\mathbf{R}}_{0}$ be the $(6 \times 6)$ extended rotation matrix:

$$
{ }^{i j} \overline{\mathbf{R}}_{0}=\left[\begin{array}{cc}
{ }^{i j} \mathbf{R}_{0} & \mathbf{0}_{3} \\
\mathbf{0}_{3} & { }^{i j} \mathbf{R}_{0}
\end{array}\right]
$$

where ${ }^{i j} \mathbf{R}_{0}$ is the rotation matrix between the global frame and the local frame attached to element $i j$, which is evaluated in the robot undeformed configuration and can be extracted using (33). Thus, multiplying ${ }^{i j} \overline{\mathbf{R}}_{0}$ by vector ${ }^{0} \mathbf{j}_{v_{i j}}^{q_{t i l}}$ yields the column $\mathbf{j}_{v_{i j}}^{q_{t i j l}}={ }^{i j} \overline{\mathbf{R}}_{0}^{0} \mathbf{j}_{v_{i j}}^{q_{t_{i j l}}}$ of Jacobian matrix $\mathbf{J}_{v_{i j}}$ defined in (28) corresponding to variable $\dot{q}_{t_{i j l}}$. 


\subsection{Stiffness and Mass Matrices of the Tree Structure}

From (6) and (9), the Lagrangian of the tree structure system can be expressed as:

$$
L_{t}=\sum_{i, j}\left(T_{i j}-V_{e_{i j}}\right)=\frac{1}{2} \sum_{i, j}\left(\left[\begin{array}{c}
\mathbf{v}_{i j}\left(A_{i j}\right) \\
\boldsymbol{\omega}_{i j}\left(A_{i j}\right) \\
\dot{\mathbf{q}}_{e_{i j}}
\end{array}\right]^{T} \mathbf{M}_{i j}\left[\begin{array}{c}
\mathbf{v}_{i j}\left(A_{i j}\right) \\
\boldsymbol{\omega}_{i j}\left(A_{i j}\right) \\
\dot{\mathbf{q}}_{e_{i j}}
\end{array}\right]-\mathbf{q}_{e_{i j}}^{T} \mathbf{K}_{i j} \mathbf{q}_{e_{i j}}\right)
$$

Introducing (28) into (39) leads to

$$
\begin{aligned}
L_{t} & =\frac{1}{2} \sum_{i, j}\left(\dot{\mathbf{q}}_{t}^{T} \mathbf{J}_{i j}^{T} \mathbf{M}_{i j} \mathbf{J}_{i j} \dot{\mathbf{q}}_{t}-\mathbf{q}_{t}^{T} \mathbf{J}_{e_{i j}}^{T} \mathbf{K}_{i j} \mathbf{J}_{e_{i j}} \mathbf{q}_{t}\right) \\
& =\frac{1}{2}\left(\dot{\mathbf{q}}_{t}^{T} \mathbf{M}_{t} \dot{\mathbf{q}}_{t}-\mathbf{q}_{t}^{T} \mathbf{K}_{t} \mathbf{q}_{t}\right)
\end{aligned}
$$

where $\mathbf{M}_{t}$ and $\mathbf{K}_{t}$ are the mass and stiffness matrices of the tree structure.

Adding the contribution of the rigid platform into (40), the Lagrangian of the total system can be written as:

$$
\begin{aligned}
L & =\frac{1}{2}\left(\dot{\mathbf{q}}_{t}^{T} \mathbf{M}_{t} \dot{\mathbf{q}}_{t}+\dot{\mathbf{x}}_{p}^{T} \mathbf{M}_{p} \dot{\mathbf{x}}_{p}-\mathbf{q}_{t}^{T} \mathbf{K}_{t} \mathbf{q}_{t}\right) \\
& =\frac{1}{2}\left(\left[\begin{array}{ll}
\dot{\mathbf{q}}_{t}^{T} & \dot{\mathbf{x}}_{p}^{T}
\end{array}\right]\left[\begin{array}{cc}
\mathbf{M}_{t} & \mathbf{0} \\
\mathbf{0} & \mathbf{M}_{p}
\end{array}\right]\left[\begin{array}{c}
\dot{\mathbf{q}}_{t} \\
\dot{\mathbf{x}}_{p}
\end{array}\right]-\left[\begin{array}{ll}
\mathbf{q}_{t}^{T} & \delta \mathbf{x}_{p}^{T}
\end{array}\right]\left[\begin{array}{cc}
\mathbf{K}_{t} & \mathbf{0} \\
\mathbf{0} & \mathbf{0}
\end{array}\right]\left[\begin{array}{c}
\mathbf{q}_{t} \\
\delta \mathbf{x}_{p}
\end{array}\right]\right) \\
& =\frac{1}{2}\left(\dot{\mathbf{q}}_{t o t}^{T} \mathbf{M}_{t o t} \dot{\mathbf{q}}_{t o t}-\mathbf{q}_{t o t}^{T} \mathbf{K}_{t o t} \mathbf{q}_{t o t}\right)
\end{aligned}
$$

where $\mathbf{M}_{p}$ is the mass matrix of the rigid platform. $\mathbf{M}_{t o t}$ and $\mathbf{K}_{t o t}$ are the total mass and stiffness matrices of the virtual system. $\mathbf{q}_{t o t}=\left[\mathbf{q}_{t}^{T} \delta \mathbf{x}_{p}^{T}\right]^{T}$ is the vector of all generalized coordinates of the virtual system.

\section{Computation of the Stiffness and Mass Matrices of the Parallel Robot}

The model of the virtual tree structure and of the free moving platform does not consider the closed-loop kinematic chains. As a matter of fact, the $n_{q_{t o t}}$ components of vector $\mathbf{q}_{t o t}$ are dependent. The independent components are gathered into vector $\mathbf{q}$ and their determination is described thereafter.

\subsection{Determination of the Generalized Coordinates of the Parallel Robot}

For determining one possible subset of generalized coordinates for the parallel robot, let us express the relations between the vector of generalized velocities $\dot{\mathbf{q}}_{t_{i}}$ and the twist of the last element $m_{i}$

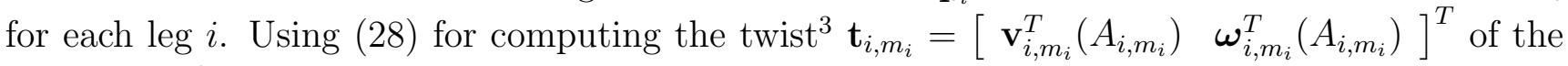
extremity of each leg, it comes:

$$
\mathbf{t}_{i, m_{i}}=\mathbf{J}_{v_{i, m_{i}}}^{i} \dot{\mathbf{q}}_{t_{i}}
$$

\footnotetext{
${ }^{3}$ Note that index $i j$ is written $i, j$ in this section for a better understanding of the equations.
} 
As the leg extremity is also linked to the rigid platform, its twist can be related to the platform twist $\dot{\mathbf{x}}_{p}$ via the rigid body displacement relation:

$$
\mathbf{t}_{i, m_{i}}=\mathbf{J}_{p}^{i} \dot{\mathbf{x}}_{p}
$$

where

$$
\mathbf{J}_{p}^{i}={ }^{i, m_{i}} \overline{\mathbf{R}}_{0}\left[\begin{array}{cc}
\mathbf{I}_{3} & {\left[\mathbf{p}_{i}\right]_{\times}} \\
\mathbf{0} & \mathbf{I}_{3}
\end{array}\right]
$$

in which $\mathbf{J}_{p}^{i}$ is a $(6 \times 6)$ matrix, $\left[\mathbf{p}_{i}\right]_{\times}$is the cross product matrix of vector $\mathbf{p}_{i}$ that characterizes the position of the attachement point $C_{i, m_{i}}$ with respect to the platform centre position (Fig. 1(a)) and ${ }^{i, m_{i}} \overline{\mathbf{R}}_{0}$ is the $(6 \times 6)$ rotation matrix between the global frame and the local frame attached to element $i, m_{i}$, evaluated in the robot undeformed configuration.

Thus, expressing the twist $\mathbf{t}_{i, m_{i}}$ for each leg as a function of the platform twist $\dot{\mathbf{x}}_{p}$ and generalized coordinates $\dot{\mathbf{q}}_{i}$, the following set of equations is obtained:

$$
\left[\begin{array}{ccc}
\mathbf{J}_{v_{1, m_{1}}}^{1} & \cdots & \mathbf{0} \\
\vdots & \ddots & \vdots \\
\mathbf{0} & \cdots & \mathbf{J}_{v_{n, m_{n}}}^{n}
\end{array}\right]\left[\begin{array}{c}
\dot{\mathbf{q}}_{t_{1}} \\
\vdots \\
\dot{\mathbf{q}}_{t_{n}}
\end{array}\right]-\left[\begin{array}{c}
\mathbf{J}_{p}^{1} \\
\vdots \\
\mathbf{J}_{p}^{n}
\end{array}\right] \dot{\mathbf{x}}_{p}=\mathbf{0}
$$

which can be equivalently written as

$$
\mathbf{J}_{v} \dot{\mathbf{q}}_{t}-\mathbf{J}_{p} \dot{\mathbf{x}}_{p}=\left[\begin{array}{ll}
\mathbf{J}_{v} & -\mathbf{J}_{p}
\end{array}\right]\left[\begin{array}{c}
\dot{\mathbf{q}}_{t} \\
\dot{\mathbf{x}}_{p}
\end{array}\right]=\mathbf{J}_{t o t} \dot{\mathbf{q}}_{t o t}=\mathbf{0}
$$

where $\mathbf{J}_{t o t}$ is a $\left(r n \times n_{q t o t}\right)$ matrix in the case of a spatial robot, $n_{q_{t o t}}>r n(r=6$ for a spatial robot, $r=3$ for a planar robot). This means that a subset $\mathbf{q}_{d}$ of $r n$ variables in vector $\mathbf{q}_{t o t}$ is linked to the others. This subset is not unique. An idea could be to put in this subset all passive joints and platform variables, i.e., $\mathbf{q}_{d}^{*}=\left[\delta \mathbf{q}_{p}^{T} \delta \mathbf{x}_{p}\right]$. However, for over-constrained parallel robots, $\operatorname{dim}\left(\mathbf{q}_{d}^{*}\right)<r n$. As a result, this vector should be completed using some other elastic variables that could be chosen arbitrarily. Meanwhile, it must be mentioned that most of parallel robots have identical legs and that such a methodology will lead to an asymmetrical description of the leg variables, which is not ideal. In order to avoid this problem, we had better put in $\mathbf{q}_{d}$ the last $r$ components $\mathbf{q}_{t_{i}}^{f}$ of each vector $\mathbf{q}_{t_{i}}$ that is now decomposed into two parts: $\mathbf{q}_{t_{i}}=\left[\mathbf{q}_{t_{i}}^{0 T} \mathbf{q}_{t_{i}}^{f T}\right]^{T}$. Thus, variables $\mathbf{q}_{t_{i}}^{f}$ are related to the others using (45):

$$
-\left[\begin{array}{ccc}
\mathbf{J}_{v_{1, m_{1}}}^{f 1} & \cdots & \mathbf{0} \\
\vdots & \ddots & \vdots \\
\mathbf{0} & \cdots & \mathbf{J}_{v_{n, m_{n}}}^{f n}
\end{array}\right]\left[\begin{array}{c}
\dot{\mathbf{q}}_{t_{1}}^{f} \\
\vdots \\
\dot{\mathbf{q}}_{t_{n}}^{f}
\end{array}\right]=\left[\begin{array}{cccc}
\mathbf{J}_{v_{1, m_{1}}}^{01} & \cdots & \mathbf{0} & -\mathbf{J}_{p}^{1} \\
\vdots & \ddots & \vdots & \vdots \\
\mathbf{0} & \cdots & \mathbf{J}_{v_{n, m_{n}}}^{0 n} & -\mathbf{J}_{p}^{n}
\end{array}\right]\left[\begin{array}{c}
\dot{\mathbf{q}}_{t_{1}}^{0} \\
\vdots \\
\dot{\mathbf{q}}_{t_{n}}^{0} \\
\dot{\mathbf{x}}_{p}
\end{array}\right]
$$

which can be rewritten as

$$
-\mathbf{J}_{v}^{f}\left[\begin{array}{c}
\dot{\mathbf{q}}_{t_{1}}^{f} \\
\vdots \\
\dot{\mathbf{q}}_{t_{n}}^{f}
\end{array}\right]=\left[\begin{array}{ll}
\mathbf{J}_{v}^{0} & -\mathbf{J}_{p}
\end{array}\right]\left[\begin{array}{c}
\dot{\mathbf{q}}_{t_{1}}^{0} \\
\vdots \\
\dot{\mathbf{q}}_{t_{n}}^{0} \\
\dot{\mathbf{x}}_{p}
\end{array}\right]
$$


or also

$$
\left[\begin{array}{c}
\dot{\mathbf{q}}_{t_{1}}^{f} \\
\vdots \\
\dot{\mathbf{q}}_{t_{n}}^{f}
\end{array}\right]=-\left(\mathbf{J}_{v}^{f}\right)^{-1}\left[\begin{array}{ll}
\mathbf{J}_{v}^{0} & -\mathbf{J}_{p}
\end{array}\right]\left[\begin{array}{c}
\dot{\mathbf{q}}_{t_{1}}^{0} \\
\vdots \\
\dot{\mathbf{q}}_{t_{n}}^{0} \\
\dot{\mathbf{x}}_{p}
\end{array}\right]=\left[\begin{array}{cccc}
\mathbf{J}_{d_{1,1}} & \cdots & \mathbf{J}_{d_{1, n}} & \mathbf{J}_{d_{1, n+1}} \\
\vdots & \ddots & \vdots & \vdots \\
\mathbf{J}_{d_{n, 1}} & \cdots & \mathbf{J}_{d_{n, n}} & \mathbf{J}_{d_{n, n+1}}
\end{array}\right] \dot{\mathbf{q}}
$$

where

- $\mathbf{J}_{v_{i, m_{i}}}^{0 i}\left(\mathbf{J}_{v_{i, m_{i}}}^{f i}\right.$, resp. $)$ collects the columns of matrix $\mathbf{J}_{v_{i, m_{i}}}^{i}$ corresponding to variables $\dot{\mathbf{q}}_{t_{i}}^{0}\left(\dot{\mathbf{q}}_{t_{i}}^{f}\right.$, resp.);

- $\mathbf{J}_{d_{i j}}$ is the matrix that maps $\dot{\mathbf{q}}_{t_{j}}^{0}$ into $\dot{\mathbf{q}}_{t_{i}}^{f}, j=1, \ldots, n$;

- $\mathbf{J}_{d_{i, n+1}}$ is the matrix that maps $\dot{\mathbf{x}}_{p}$ into $\dot{\mathbf{q}}_{t_{i}}^{f}$.

It is noteworthy that the inversion of matrix $\mathbf{J}_{v}^{f}$ involves only the inversion of the $(r \times r)$ matrices $\mathbf{J}_{v_{i, m_{i}}}^{f i}$, which is more efficient in terms of computational time. Moreover, when 3D beam elements are used for leg $i$, if the coordinates $\mathbf{q}_{t_{i}}^{f}$ are the elastic coordinates of $l$-th element of this leg (previesly denoted as $\mathbf{q} \mathbf{e}_{i, l}$ ), it can be proven that, as the $k$-th column of matrix $\mathbf{J}_{v_{i l}}^{f i}$ corresponds to a unit twist that describes the displacement of the leg extremity due to the $k$-th coordinate of vector $\mathbf{q}_{t_{i}}^{f}, \mathbf{J}_{v_{i l}}^{f i}$ is equal to [23]:

$$
\mathbf{J}_{v_{i l}}^{f i}=\left[\begin{array}{cc}
i, m_{i} & \mathbf{R}_{i l}{ }^{i, m_{i}} \mathbf{R}_{i l}\left[\mathbf{p}_{i l}\right]_{\times} \\
\mathbf{0} & { }^{i, m_{i}} \mathbf{R}_{i l}
\end{array}\right]
$$

where ${ }^{i, m_{i}} \mathbf{R}_{i l}$ is the rotation matrix between the local frame linked at element $i, m_{i}$ and the local frame linked to element $i l$ and $\left[\mathbf{p}_{i l}\right]_{\times}$is the cross product matrix of the vector $\mathbf{p}_{i l}$ that characterizes the position of the leg extremity with respect to the frame linked to element $i l$. Thus, its matrix inverse is equal to

$$
\left(\mathbf{J}_{v_{i l}}^{f i}\right)^{-1}=\left[\begin{array}{cc}
{ }^{i, m_{i}} \mathbf{R}_{i l}^{T} & -\left(\left[\mathbf{p}_{i l}\right]_{\times}{ }^{i, m_{i}} \mathbf{R}_{i l}^{T}\right) \\
\mathbf{0} & { }_{i, m_{i}} \mathbf{R}_{i l}^{T}
\end{array}\right]
$$

which requires few calculations and, before all, avoid any numerical inversion that could lead to numerical issues. If $2 \mathrm{D}$ beam elements are used, some similar relations can be obtained.

Finally, the Jacobian matrix relating all variables $\dot{\mathbf{q}}_{t o t}$ to the independent variables $\dot{\mathbf{q}}=$ $\left[\dot{\mathbf{q}}_{t_{1}}^{0 T} \cdots \dot{\mathbf{q}}_{t_{n}}^{0 T} \dot{\mathbf{x}}_{p}\right]^{T}$ can be obtained as:

$$
\dot{\mathbf{q}}_{t o t}=\left[\begin{array}{c}
\dot{\mathbf{q}}_{t_{1}}^{0} \\
\dot{\mathbf{q}}_{t_{1}}^{f} \\
\vdots \\
\dot{\mathbf{q}}_{t_{n}}^{0} \\
\dot{\mathbf{q}}_{t_{n}}^{f} \\
\dot{\mathbf{x}}_{p}
\end{array}\right]=\left[\begin{array}{cccc}
\mathbf{I}_{c_{1}} & \cdots & \mathbf{0} & \mathbf{0} \\
\mathbf{J}_{d_{1,1}} & \cdots & \mathbf{J}_{d_{1, n}} & \mathbf{J}_{d_{1, n+1}} \\
\vdots & \cdots & \vdots & \vdots \\
\mathbf{0} & \cdots & \mathbf{I}_{c_{n}} & \mathbf{0} \\
\mathbf{J}_{d_{n, 1}} & \cdots & \mathbf{J}_{d_{n, n}} & \mathbf{J}_{d_{n, n+1}} \\
\mathbf{0} & \cdots & \mathbf{0} & \mathbf{I}_{6}
\end{array}\right]\left[\begin{array}{c}
\dot{\mathbf{q}}_{t_{1}}^{0} \\
\vdots \\
\dot{\mathbf{q}}_{t_{n}}^{0} \\
\dot{\mathbf{x}}_{p}
\end{array}\right]=\mathbf{J} \dot{\mathbf{q}}
$$

where $\mathbf{I}_{c_{i}}$ is the $\left(c_{i} \times c_{i}\right)$ identity matrix, $c_{i}$ being the dimension of vector $\dot{\mathbf{q}}_{t_{i}}^{0}$. Under the assumption of small displacements, the following relation holds true:

$$
\mathbf{q}_{t o t}=\mathbf{J q}
$$




\subsection{Computation of the Natural Frequencies of the Parallel Robot}

Introducing (52) and (53) into (41) leads to:

$$
L=\frac{1}{2}\left(\dot{\mathbf{q}}^{T} \mathbf{J}^{T} \mathbf{M}_{t o t} \mathbf{J} \dot{\mathbf{q}}-\mathbf{q}^{T} \mathbf{J}^{T} \mathbf{K}_{t o t} \mathbf{J} \mathbf{q}\right)=\frac{1}{2}\left(\dot{\mathbf{q}}^{T} \mathbf{M} \dot{\mathbf{q}}-\mathbf{q}^{T} \mathbf{K} \mathbf{q}\right)
$$

Since in the natural frequency problem, matrices $\mathbf{M}$ and $\mathbf{K}$ are evaluated in the robot undeformed configuration, namely, for $\mathbf{q}_{e}=\mathbf{0}$, and as a result for $\delta \mathbf{q}_{a}=\mathbf{0}$ and $\delta \mathbf{q}_{p}=\mathbf{0}$, it turns out that the Lagrange equations yield:

$$
\frac{d}{d t}\left(\frac{\partial L}{\partial \dot{\mathbf{q}}}\right)-\frac{\partial L}{\partial \mathbf{q}}=\mathbf{M} \ddot{\mathbf{q}}+\mathbf{K q}=\mathbf{0}
$$

A solution $\mathbf{q}_{l}$ of this equation satisfies:

$$
\left(\omega_{l}^{2} \mathbf{M}-\mathbf{K}\right) \mathbf{q}_{l}=\mathbf{0}
$$

where $\omega_{l}=2 \pi f_{l}, f_{l}$ is the natural frequency associated with the $l$-th natural mode of vibrations and $\mathbf{q}_{l}$ is its associated eigenvector.

Therefore, the natural frequencies of the parallel robot are evaluated by solving the following eigenvalue problem:

$$
\operatorname{det}\left(\omega_{l}^{2} \mathbf{M}-\mathbf{K}\right)=0
$$

In the next section, the natural frequencies of the NaVARo, a parallel robot developed at IRCCyN [17], are computed using the proposed method and compared with the results obtained with the Cast3M software [19] and experimentally.

\section{Case Study: Computation of the Natural Frequencies of the NaVaRo}

\subsection{Description of the NaVARo}

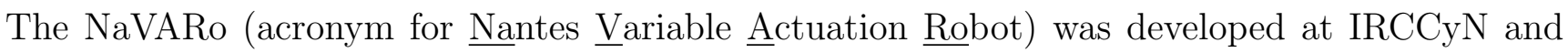
is shown in Fig. 3(a). The NaVARo is a 3-dof planar parallel manipulator composed of three identical legs and one moving platform made up of three segments $E_{1} P, E_{2} P$ and $E_{3} P$ rigidly linked at point $P$. The $i$-th leg contains four links $A_{i} B_{i}, B_{i} C_{i}, C_{i} E_{i}, A_{i} D_{i}$ (named link $2 i$, link $3 i$, link $4 i$ and link $1 i$, respectively) connected with five revolute joints in such a way that $A_{i} B_{i} C_{i} D_{i}$ is a parallelogram linkage, $i=1,2,3$. The base frame $\mathcal{F}_{b}\left(O, \mathbf{x}_{0}, \mathbf{y}_{0}, \mathbf{z}_{0}\right)$ (not shown in Fig. $3(\mathrm{~b})$ ) is defined such as point $O$ is located at the geometric centre of the equilateral triangle $A_{1} A_{2} A_{3}$. Frame $\mathcal{F}_{p}\left(P, \mathbf{x}_{p}, \mathbf{y}_{p}, \mathbf{z}_{p}\right)$ is attached to the moving platform. In the home configuration shown in Fig. $3, \mathcal{F}_{b}$ and $\mathcal{F}_{p}$ coincide. $\left(x_{p}, y_{p}\right)$ are the Cartesian coordinates of point $P$ expressed in frame $\mathcal{F}_{b}$ and $\theta_{p}$ is the orientation angle of the moving platform, namely, the angle between $\mathbf{x}_{0}$ and $\mathbf{x}_{p}$.

$q_{1 i}$ denotes the angle between axis $\mathbf{x}_{0}$ and link $1 i . q_{2 i}$ denotes the angle between link $1 i$ and link $2 i$. Three double clutches are mounted to the base and located at points $A_{i}, i=1,2,3$, in order to actuate either angle $q_{1 i}$ or angle $q_{2 i}$. As a consequence, the NaVARo has eight actuation modes as described in $[17,24]$. Therefore, the moving platform can be moved throughout the 


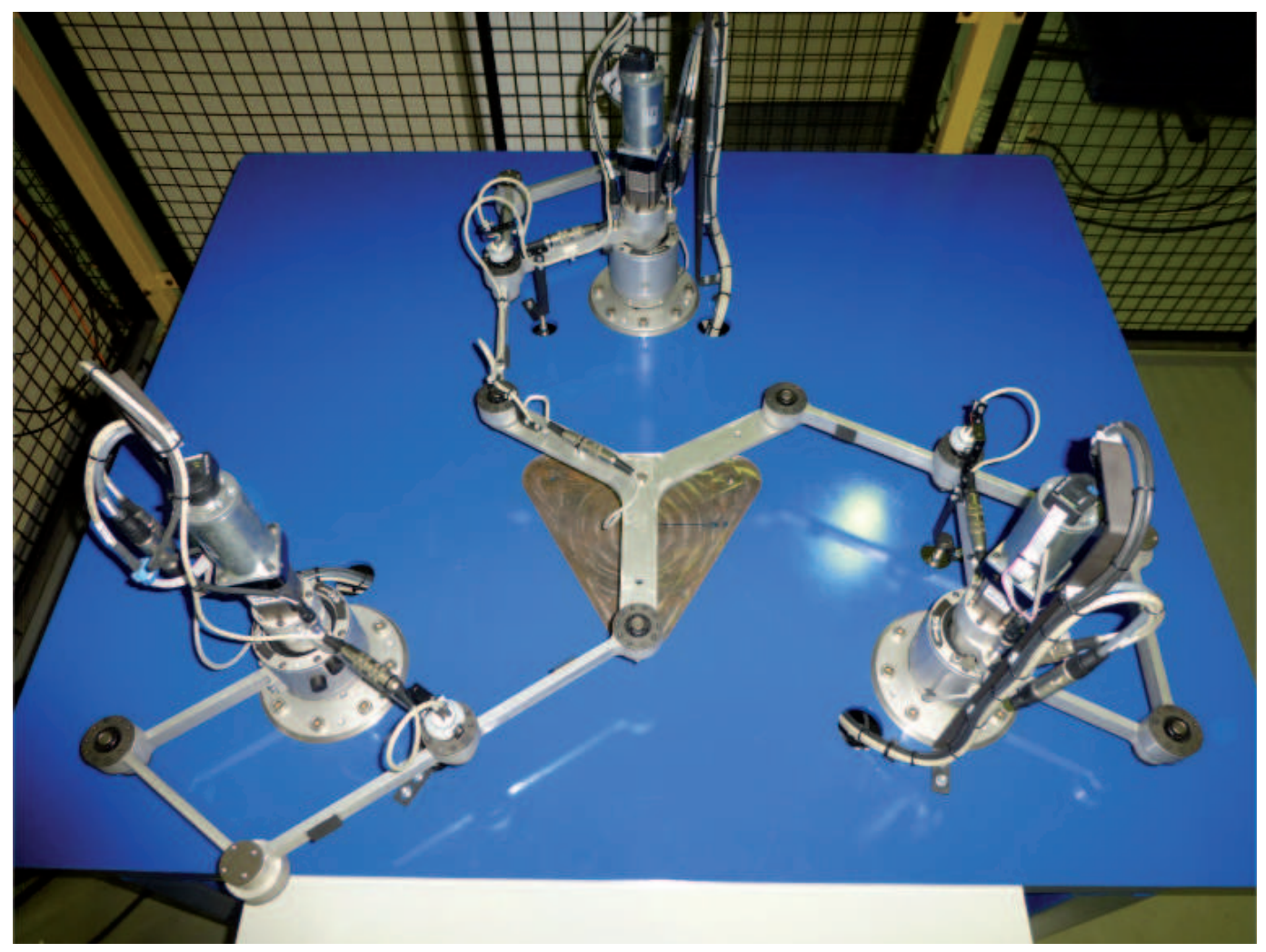

(a) Prototype of the NaVARo located at IRCCyN, Nantes, France

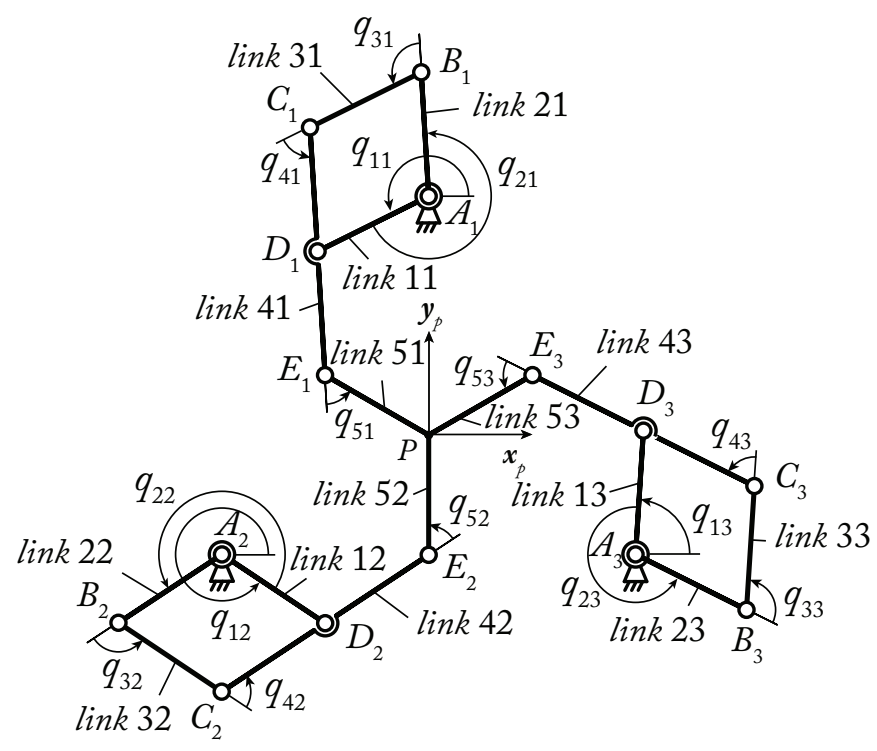

(b) Shematics of the NaVARo

Figure 3: The NaVARo 
manipulator workspace without reaching any parallel singularity thanks to a judicious actuation scheme.

The kinematics of the $i$-th leg is described by the modified Denavit-Hartenberg parameters $(M D H)$ [23] given in Table 1 , in which $\gamma_{i}=\pi / 2$ if $i=1, \gamma_{i}=-5 \pi / 6$ if $i=2$ and $\gamma_{i}=-\pi / 6$ if $i=3$. Besides, the circumradius of the moving-platform is equal to $0.2027 \mathrm{~m}$, i.e., $l_{5 i}=0.2027$ $\mathrm{m}$.

Table 1: $M D H$ parameters of the $i$-th leg

\begin{tabular}{ccccccccc}
\hline \hline$j i$ & $a(j i)$ & $\sigma_{j i}$ & $\gamma_{j i}$ & $b_{j i}$ & $\alpha_{j i}$ & $d_{j i}$ & $\theta_{j i}$ & $r_{j i}$ \\
\hline $1 i$ & 0 & 0 & $\gamma_{i}$ & 0 & 0 & $d_{1}=0.4041 \mathrm{~m}$ & $q_{1 i}-\gamma_{i}$ & 0 \\
$2 i$ & 0 & 0 & $\gamma_{i}$ & 0 & 0 & $d_{1}=0.4041 \mathrm{~m}$ & $q_{2 i}-\gamma_{i}$ & 0 \\
$3 i$ & $2 i$ & 0 & 0 & 0 & 0 & $d_{3}=0.2100 \mathrm{~m}$ & $q_{3 i}$ & 0 \\
$4 i$ & $3 i$ & 0 & 0 & 0 & 0 & $d_{4}=0.2100 \mathrm{~m}$ & $q_{4 i}$ & 0 \\
$5 i$ & $4 i$ & 0 & 0 & 0 & 0 & $d_{5}=0.4200 \mathrm{~m}$ & $q_{5 i}$ & 0 \\
\hline \hline
\end{tabular}

Each link, of rectangular cross-section, is made up of duraluminum alloy $(E=74000 \mathrm{MPa}$, $G=28900 \mathrm{MPa}, \rho=2800 \mathrm{~kg} / \mathrm{m}^{3}$ ). Table 2 gives the cross-section area and the moments of inertia of the robot links.

Table 2: Characteristics of the beam cross-sections

\begin{tabular}{rrrrrr}
\hline \hline link & $A_{i j}\left(\mathrm{~m}^{2}\right)$ & $I_{y_{i j}}\left(\mathrm{~m}^{4}\right)$ & $I_{z_{i j}}\left(\mathrm{~m}^{4}\right)$ & $I_{p_{i j}}\left(\mathrm{~m}^{4}\right)$ & $I_{0_{i j}}\left(\mathrm{~m}^{4}\right)$ \\
\hline $1 i, 2 i, 3 i, 4 i$ & $2.4 \cdot 10^{-4}$ & $1.152 \cdot 10^{-8}$ & $2.000 \cdot 10^{-9}$ & $1.352 \cdot 10^{-8}$ & $5.902 \cdot 10^{-9}$ \\
$5 i$ & $4 \cdot 10^{-4}$ & $3.333 \cdot 10^{-8}$ & $5.333 \cdot 10^{-8}$ & $8.666 \cdot 10^{-8}$ & $1.123 \cdot 10^{-8}$ \\
\hline \hline
\end{tabular}

In the experimental setup, the rotation of links $1 i$ and $2 i$ about point $A_{i}, i=1,2,3$, is locked thanks to the double clutch mechanisms. The elasto-dynamic modeling of the NaVARo is complex due to the closed-loop chain in each leg and is obtained by following those three steps:

1. Computation of the mass and stiffness matrices of the virtual system assuming that the moving platform is cut at point $P$ and the parallel linkages are opened at points $D_{i}, i=$ $1,2,3$;

2. Computation of the mass and stiffness matrices of the legs including the closed-loop chains;

3. Computation of the mass and stiffness matrices of the NaVARo.

A single 3D beam element is used to model links $1 i, 2 i, 3 i$ and $5 i$ (see Section 2.4) while two $3 \mathrm{D}$ beam elements of equal lengths $l\left(l=l_{C_{i} D_{i}}=l_{D_{i} E_{i}}\right)$ are used to model links $4 i$. Decomposing links $4 i$ into two beam elements is mandatory in order to be able to close the leg loops as mentioned previously in the step 2. Thus, the NaVARo is modelled as a spatial mechanism and its elastodynamic model contains 144 generalized coordinates: (i) 108 elastic coordinates; (ii) 12 passive joint coordinates, i.e., four passive joint angles per leg; (iii) 18 intermediary coordinates for the assembly of the legs; (iv) 6 coordinates for the moving-platform pose. From Sec. 4, it turns out that there are only 90 independent coordinates amongst those 144 coordinates. 


\subsection{Numerical analysis}

A Matlab code was written to compute the robot mass and stiffness matrices using the modeling procedure presented in Secs. 2 to 4 . The obtained robot mass and stiffness matrices were validated by means of an equivalent model developed using Cast3M software [19]. Cast3M aims to determine the elastodynamic model of structures modeled with beams. Both models give the same values for the first 90 natural frequencies of the NaVARo. Table 3 gives the first five natural frequencies of the NaVARo for the eight robot postures shown in Fig. 4.

The natural frequencies of the NaVARo are the same for poses 3, 5 and 7 (4, 6 and 8, resp.) as they correspond to a rotation of the robot base frame of $\pm 120 \mathrm{deg}$ with respect to pose 3 (pose 4 , resp.).

Table 3: Comparison of the natural frequencies obtained with Cast3M and the Matlab model.

\begin{tabular}{ccccccccc}
\hline \hline$(\mathrm{Hz})$ & Pose 1 & Pose 2 & Pose 3 & Pose 4 & Pose 5 & Pose 6 & Pose 7 & Pose 8 \\
\hline$f_{1}($ Cast3M) & 44.10 & 45.71 & 36.98 & 40.17 & 36.98 & 40.17 & 36.98 & 40.17 \\
$f_{1}($ Matlab model $)$ & 44.10 & 45.71 & 36.98 & 40.17 & 36.98 & 40.17 & 36.98 & 40.17 \\
$f_{2}($ Cast3M) & 44.10 & 45.71 & 49.31 & 50.32 & 49.31 & 50.32 & 49.31 & 50.32 \\
$f_{2}$ (Matlab model $)$ & 44.10 & 45.71 & 49.31 & 50.32 & 49.31 & 50.32 & 49.31 & 50.32 \\
$f_{3}($ Cast3M) & 53.98 & 54.58 & 53.37 & 52.99 & 53.37 & 52.99 & 53.37 & 52.99 \\
$f_{3}$ (Matlab model) & 53.98 & 54.58 & 53.37 & 52.99 & 53.37 & 52.99 & 53.37 & 52.99 \\
$f_{4}($ Cast3M) & 60.63 & 65.35 & 67.28 & 67.36 & 67.28 & 67.36 & 67.28 & 67.36 \\
$f_{4}$ (Matlab model $)$ & 60.63 & 65.35 & 67.28 & 67.36 & 67.28 & 67.36 & 67.28 & 67.36 \\
$f_{5}($ Cast3M) & 95.62 & 97.92 & 91.80 & 91.52 & 91.80 & 91.52 & 91.80 & 91.52 \\
$f_{5}$ (Matlab model $)$ & 95.62 & 97.92 & 91.80 & 91.52 & 91.80 & 91.52 & 91.80 & 91.52 \\
\hline \hline
\end{tabular}

\subsection{Experiments}

Some experimental tests were carried out using the setup presented in Fig. 5. The application of experimental modal testing to the NaVARo was done through impact hammer excitation, a 3-D accelerometer response and data postprocessing, conducted using the DataBox software developed at IRCCyN and sold by MITIS company. The points and directions of excitation were chosen on points $B_{i}$ and $E_{i}$ of each leg along all axes in order to get the maximal number of resonance frequencies. Piezoelectric triaxial accelerometers with a sensitivity of $1000 \mathrm{mV} / \mathrm{g}$ were used to pick up the three acceleration responses. The acquisitions were performed for the eight robot postures shown in Fig. 4. Each measurement resolution is equal to $1 \mathrm{~Hz}$ as the acquisition time and the sampling time are equal to $1 \mathrm{~s}$ and $40 \mu \mathrm{s}$, respectively.

The resonance frequencies were obtained with a fast Fourier transform of the signals given by the triaxial accelerometer. As a result, the measured resonance frequencies between 0 and $80 \mathrm{~Hz}$ for poses 1 to 4 are given in Table 4. As the results for poses 3, 5 and 7 (poses 4, 6 and 8, resp.) are similar due to the manipulator symmetry, only the results for poses 3 and 4 are given in Table 4 and the redundant poses were used to highlight some resonance frequencies with low energy level.

It is noteworthy that the resonance frequencies of the NaVARo amount to its natural frequencies as the damping is supposed to be negligible. 
Table 4: NaVARo natural frequencies (measured and computed using refined model) between 0 and $80 \mathrm{~Hz}$.

\begin{tabular}{ccccc}
\hline \hline$(\mathrm{Hz})$ & Pose 1 & Pose 2 & Pose 3 & Pose 4 \\
\hline$f_{1}$ meas. & 22 & 19 & 17 & 18 \\
$f_{1}$ calc. & 19.25 & 19.46 & 17.91 & 18.44 \\
$f_{2}$ meas. & 24 & 21 & 19 & 20 \\
$f_{2}$ calc. & 20.43 & 20.49 & 19.71 & 19.26 \\
$f_{3}$ meas. & 32 & - & 23 & 22 \\
$f_{3}$ calc. & 40.25 & 41.88 & 20.91 & 21.28 \\
$f_{4}$ meas. & - & 44 & 27 & 33 \\
$f_{4}$ calc. & 43.16 & 45.55 & - & 36.88 \\
$f_{5}$ meas. & 42 & 45 & 32 & 43 \\
$f_{5}$ calc. & 44.10 & 47.05 & 36.88 & 40.60 \\
$f_{6}$ meas. & 50 & 53 & 43 & 44 \\
$f_{6}$ calc. & - & - & 41.86 & 46.13 \\
$f_{7}$ meas. & 52 & 54 & 46 & 50 \\
$f_{7}$ calc. & - & 56.37 & 45.61 & 55.29 \\
$f_{8}$ meas. & 62 & 56 & 48 & 56 \\
$f_{8}$ calc. & 67.94 & - & 50.52 & 57.81 \\
$f_{9}$ meas. & 66 & 60 & 57 & 58 \\
$f_{9}$ calc. & 68.81 & 63.10 & 55.45 & 62.27 \\
$f_{10}$ meas. & 77 & - & 60 & 66 \\
$f_{10}$ calc. & 79.79 & - & 61.04 & - \\
$f_{11}$ meas. & - & - & 61 & - \\
$f_{11}$ calc. & - & - & - & - \\
$f_{12}$ meas. & - & - & 65 & - \\
$f_{12}$ calc. & - & - & 65.00 & - \\
\hline \hline
\end{tabular}




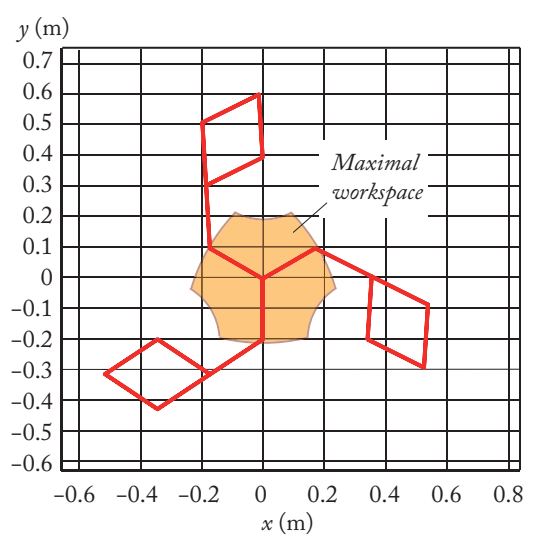

(a) Pose $1 x=0 \mathrm{~m}, y=0 \mathrm{~m}$, $\theta=0 \mathrm{rad}$

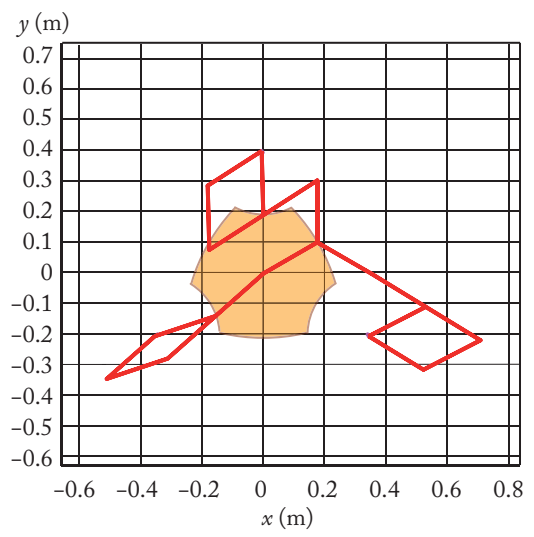

(d) Pose $4 x=0.182 \mathrm{~m}, y=$ $0.105 \mathrm{~m}, \theta=-\pi / 3 \mathrm{rad}$

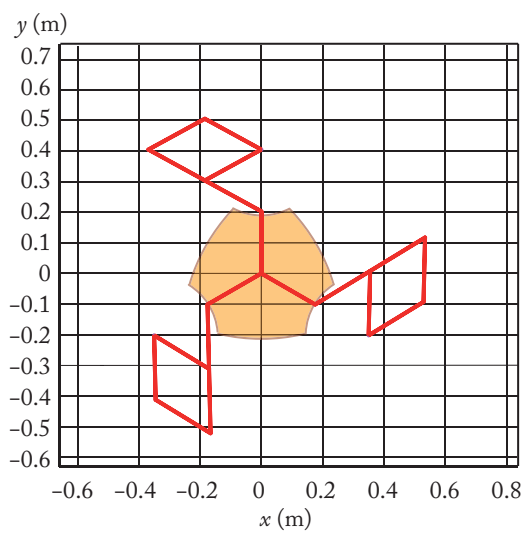

(b) Pose $2 x=0 \mathrm{~m}, y=0 \mathrm{~m}$, $\theta=-\pi / 3 \mathrm{rad}$

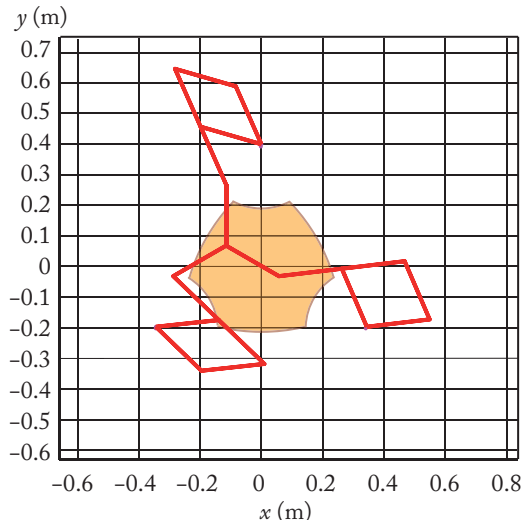

(e) Pose $5 x=-0.117 \mathrm{~m}, y=$ $0.068 \mathrm{~m}, \theta=-\pi / 3 \mathrm{rad}$

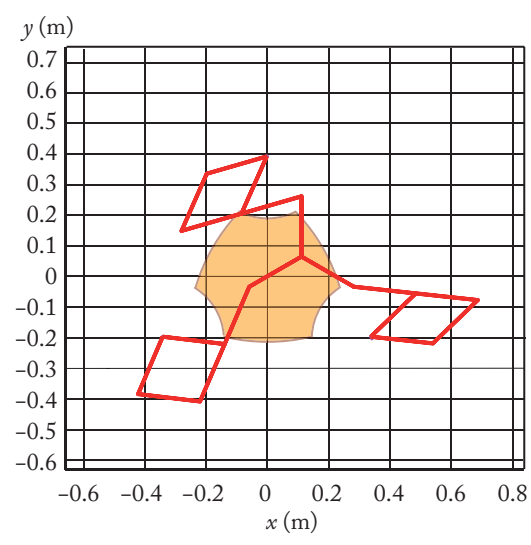

(c) Pose $3 x=0.117 \mathrm{~m}, y=$ $0.068 \mathrm{~m}, \theta=-\pi / 3 \mathrm{rad}$

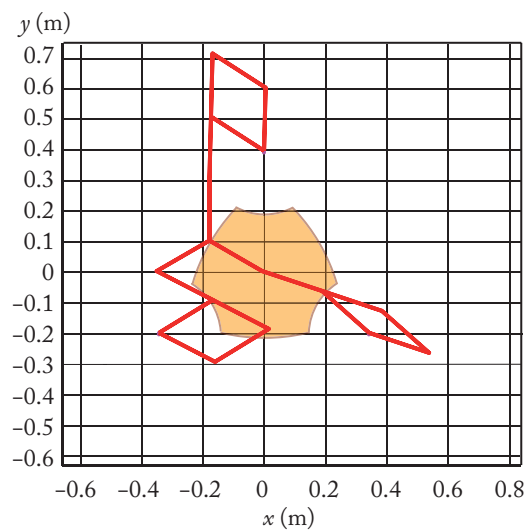

(f) Pose $6 x=-0.182 \mathrm{~m}, y=$ $0.105 \mathrm{~m}, \theta=-\pi / 3 \mathrm{rad}$

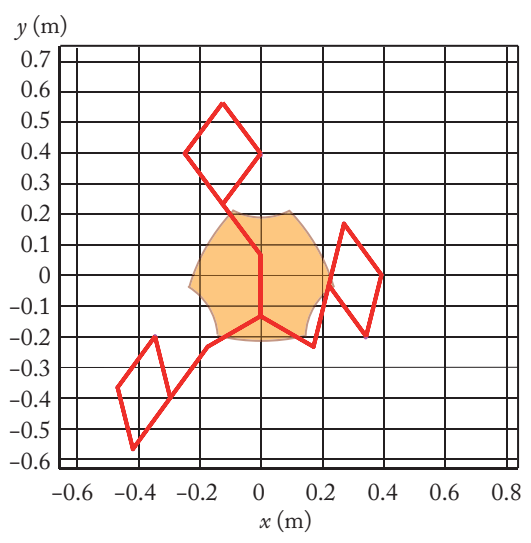

(g) Pose $7 x=0 \mathrm{~m}, y=-0.135 \mathrm{~m}$, $\theta=-\pi / 3 \mathrm{rad}$

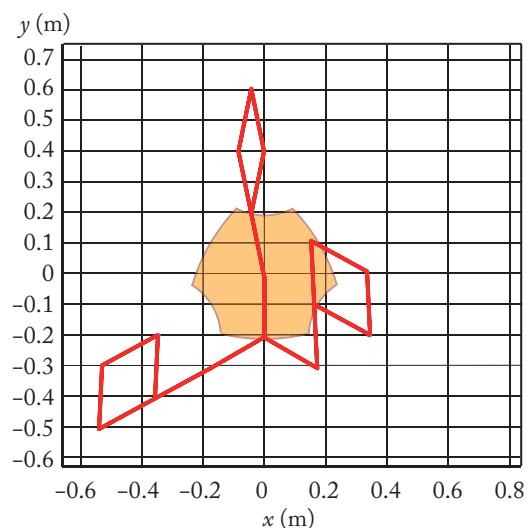

(h) Pose $8 x=0 \mathrm{~m}, y=-0.21 \mathrm{~m}$, $\theta=-\pi / 3 \mathrm{rad}$

Figure 4: The eight poses used for the experiments

It is apparent that the results given Table 4 do not match with those shown in Table 3 . As a matter of fact, the elasticity of the clutches has not been modelled and the joint masses have 


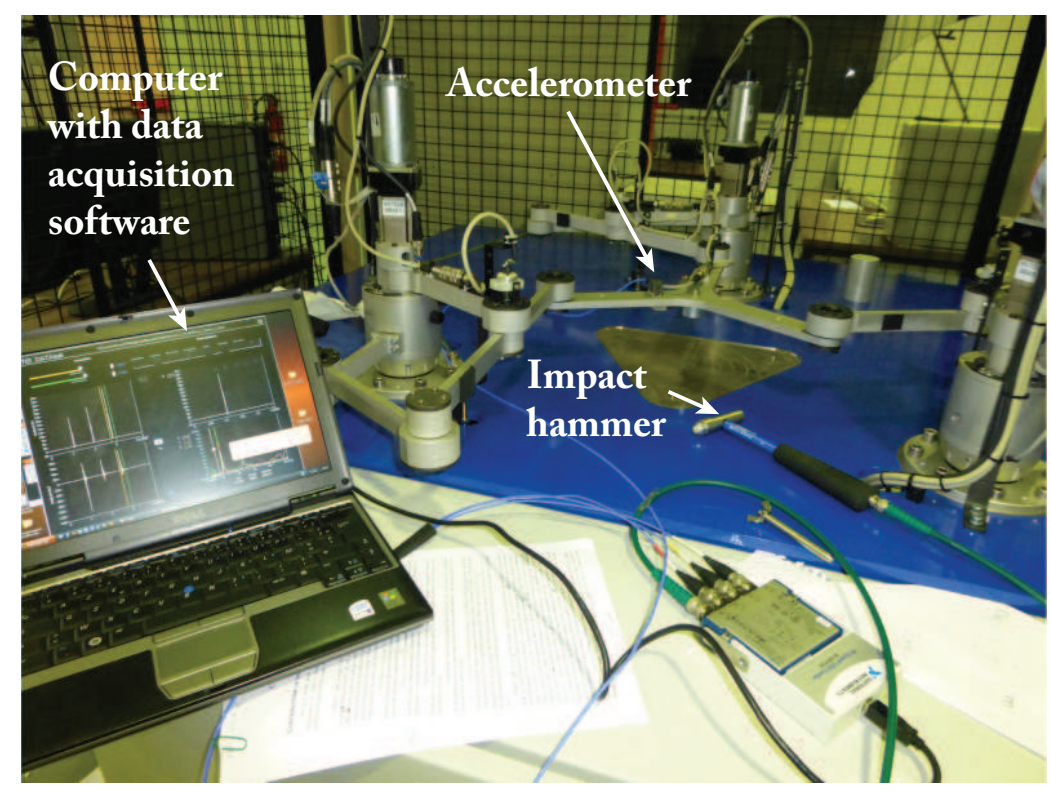

Figure 5: Experimental setup: DataBox

been omitted with Cast3M software as the latter cannot model lumped masses. Thus, a refined Matlab model was written in order to consider joint masses (about $300 \mathrm{~g}$ per joint) and elasticities in clutches (about $2000 \mathrm{Nm} / \mathrm{rad}$ ).

Thus, the natural frequencies of the NaVARo computed with this refined model and the measured frequencies are gathered in Table 4 by comparing the computed mode shapes with the hammer impact direction and the direction of the vibration signals, the latter being measured by the triaxial accelerometer.

We can notice that there is a good correlation between the measured frequencies and the computed natural frequencies.

Nevertheless, few predicted frequencies do not match with the measurements and vice-versa. Indeed, the theoretical and experimental results may differ due to the following reasons:

- The NaVARo has not been calibrated yet and there are some errors in the estimated moving platform pose;

- The passive joint elasticity has not been considered;

- The robot links are supposed to be coplanar in the theoretical model, whereas they are not in the prototype for collision avoidance;

- The robot links are not perfect beams as both ends are widened to insert ball bearings;

- The theoretical elastodynamic model does not consider any damping effect.

However, from those experiments, we can claim that the theoretical model is satisfactory and the proposed modeling procedure is efficient for reproducing the real behavior of any parallel robot. 


\section{Conclusion}

Parallel robots have been increasingly used in the industry in the last few years and the characterization of their elastodynamic behaviour is still an issue. Accurate elastodynamic models of parallel robots are useful at both their design and control stages in order to define their optimal dimensions and shapes while improving their vibratory behaviour. Several models have been proposed in the literature. However, even if they can be adapted to any type of mechanism, they are not directly devoted to parallel manipulators and they do not provide a systematic and straightforward way for computing the Jacobian matrices associated with the kinematic constraints. Moreover, they do not take into account the symmetry in the robot leg description for choosing the independent coordinates describing the robot motion.

Therefore, a systematic method for the natural frequency computation of parallel robots has been developed in this paper. Indeed, the Jacobian matrices related to the kinematic constraints of the parallel robots are obtained in a straightforward way. Moreover, a way of choosing a symmetrical set of leg variables has been proposed. Contrary to most of the existing methods, the proposed approach does not contain any numerical matrix inversion, which is better to avoid numerical issues that may lead to a loss in the result accuracy.

This proposed approach has been used to compute the natural frequencies of the NaVARo, which is a planar parallel manipulator with multiple actuation modes developed at IRCCyN. The foregoing computed natural frequencies and those obtained with Cast3M software by using an equivalent robot model turned out to be identical. Then, some experiments have been carried out through impact hammer excitation and measurements of the platform displacements with a 3D accelerometer. The resonance frequencies obtained with a fast Fourier transform of the signals given by the triaxial accelerometer have been compared with the frequencies computed from a refined model of the robot. It appeared that there is a good correlation between the natural frequencies of the NaVARo computed with this refined model and the measured excitation frequencies.

\section{Acknowledgements}

This work has been partially funded by the French ANR project ARROW (ANR 2011 BS3 006 01). The authors would like to thank the MO2P team of IRCCyN for the impact hammer and accelerometers loan. Dr. Mathieu Ritou and Mr. Jean-Pierre Regoin are also acknowledged for their great help and good advice during the experiments on the NaVARo.

\section{References}

[1] T. Brogardh. Present and future robot control development an industrial perspective. Annual Reviews in Control, 31(1):69-79, 2007.

[2] H. Chanal, E. Duc, and P. Ray. A study of the impact of machine tool structure on machining processes. International Journal of Machine Tools and Manufacture, 46(2):98-106, 2006. 
[3] P.A. Voglewede and I. Ebert-Uphoff. Overatching framework for measuring the closeness to singularities of parallel manipulators. IEEE Transactions on Robotics, 21(6):1037-1045, 2005.

[4] B. C. Bouzgarrou, P. Ray, and G. Gogu. New approach for dynamic modelling of flexible manipulators. Part K: Journal of Multi-body Dynamics, 219(3):285-298, 2005.

[5] S. Briot, A. Pashkevich, and D. Chablat. On the optimal design of parallel robots taking into account their deformations and natural frequencies. In Proceedings of the ASME 2009 International Design Engineering Technical Conferences $\&$ Computers and Information in Engineering Conference IDETC/CIE, San Diego, California, 2009.

[6] N. C. Singer and W. P. Seering. Preshaping Command Inputs to Reduce System Vibration. Massachusetts Institute of Technology Artificial Intelligence Laboratory, January 1988. A.I. Memo No. 1027.

[7] T. Singh and W. E. Singhose. Tutorial on input shaping/time delay control of maneuvering flexible structures. In American Control Conference, May 2002.

[8] G. Pelaez, Gu. Pelaez, J.M. Perez, A. Vizan, and E. Bautista. Input shaping reference commands for trajectory following cartesian machines. Control Engineering Practice, 13(8):941 - 958, 2005. Fault Detection, Supervision and Safety of Technical Processes (Safeprocess 2003).

[9] B.C. Bouzgarrou, J.C. Fauroux, G. Gogu, and Y. Heerah. Rigidity analysis of t3r1 parallel robot uncoupled kinematics. In Proceedings of the 35th International Symposium on Robotics, Paris, France, 2004.

[10] F. Bettaieb, P. Cosson, and J.-Y. Hascot. Modeling of a high-speed machining center with a multibody approach: the dynamic modeling of flexible manipulators. In Proceedings of the 6th International Conference on High Speed Machining, San Sebastian, Spain, 2007.

[11] A. Shabana. Dynamics of Multibody Systems. Cambridge University Press, 2005.

[12] M. Rognant, E. Courteille, and P. Maurine. A systematic procedure for the elastodynamic modeling and identification of robot manipulators. IEEE Transactions on Robotics, 26(6):1085-1093, 2010.

[13] O.A. Bauchau. Flexible multibody dynamics. Springer, 2011.

[14] A. Cammarata, D. Condorelli, and R. Sinatra. An algorithm to study the elastodynamics of parallel kinematic machines with lower kinematic pairs. ASME Transactions Journal of Mechanisms and Robotics, 5(1), 2013.

[15] E. Wittbrodt, I. Adamiec-Wójcik, and S. Wojciech. Dynamics of Flexible Multibody Systems. Springer-Verlag Berlin Heidelberg, 2006.

[16] O. Ibrahim and W. Khalil. Inverse and direct dynamic models of hybrid robots. Mechanism and Machine Theory, 45:627-640, 2010. 
[17] N. Rakotomanga, D. Chablat, and S. Caro. Kinetostatic performance of a planar parallel mechanism with variable actuation. In Advances in Robot Kinematics, pages 311-320, Batzsur-Mer, France, June 2008.

[18] S. Caro, D. Chablat, P. Wenger, and X Kong. Kinematic and dynamic modeling of a parallel manipulator with eight actuation modes. New Trends in Medical and Service Robots, Springer, pages 315-329, 2014.

[19] Castem3000. The castem software webpage. www-cast3m.cea.fr. Webpage accessed November 2012 .

[20] F. Boyer and W. Khalil. Dynamics of a 3-dof spatial parallel manipulator with flexible links. In IEEE International Conference on Robotics and Automation, 1996. Proceedings., 1995 IEEE International Conference on, pages 1947-1952, April 1996.

[21] R.D. Blevins. Formulas for natural frequency and mode shape. Krieger Publishing Company, 2001.

[22] A. Pashkevich, D. Chablat, and P. Wenger. Stiffness analysis of overconstrained parallel manipulators. Mechanism and Machine Theory, 44(5):966-982, 2009.

[23] W. Khalil and E. Dombre. Modeling, Identification and Control of Robots. Hermes Penton London, 2002.

[24] V. Arakelian, S. Briot, and V. Glazunov. Increase of singularity-free zones in the workspace of parallel manipulators using mechanisms of variable structure. Mechanism and Machine Theory, 43(9):1129-1140, 2008. 